\title{
CHARACTERIZATION OF SHAPE MEMORY ALLOYS USING ARTIFICIAL NEURAL NETWORKS
}

\author{
A Thesis \\ by \\ JAMES VICTOR HENRICKSON III
}

\begin{abstract}
Submitted to the Office of Graduate and Professional Studies of Texas A\&M University in partial fulfillment of the requirements for the degree of

MASTER OF SCIENCE
\end{abstract}

Chair of Committee, John Valasek

Committee Members, Thomas W. Strganac

Arun Srinivasa

Head of Department, Rodney D.W. Bowersox

May 2014

Major Subject: Aerospace Engineering

Copyright 2014 James Victor Henrickson III 


\begin{abstract}
Shape memory alloys are capable of delivering advantageous solutions to a wide range of engineering-based problems. Implementation of these solutions, however, is often complicated by the hysteretic, non-linear, thermomechanical behavior of the material. Existing constitutive models are largely capable of accurately describing this unique behavior, but they require prior characterization of material parameters. Current characterization procedures necessitate extensive data collection and data processing, creating a high barrier of entry for shape memory alloy application. This thesis develops a novel approach in which a form of computational intelligence is applied to the task of shape memory alloy material parameter characterization. Specifically, this work develops a methodology in which an artificial neural network is trained to identify transformation temperatures and stress influence coefficients of shape memory alloy specimens using strain-temperature coordinates as inputs. Training data is generated through the use of an existing shape memory alloy constitutive model. Factorial and Taguchi-based methods of generating training data are implemented and compared. Results show that trained artificial neural networks are capable of identifying shape memory alloy material parameters with satisfactory accuracy. Comparison of the implemented training data generation methods indicates that the Taguchi-based approach yields an artificial neural network that outperforms that of the factorial-based approach despite requiring significantly fewer training data specimens.
\end{abstract}




\section{ACKNOWLEDGEMENTS}

Funding was provided by the National Science Foundation through the national Research Experience for Undergraduates program. This support is gratefully acknowledged by the author. In addition to his committee chair and members - Dr. John Valasek, Dr. Arun Srinivasa, and Dr. Thomas Strganac - the author would further like to thank Dr. Kenton Kirkpatrick and Dr. Darren Hartl for many insightful discussions and comments during the course of this work. 


\section{NOMENCLATURE}

\begin{tabular}{|c|c|}
\hline$\alpha$ & Thermal coefficient of expansion $\left(1 /{ }^{\circ} \mathrm{C}\right)$ \\
\hline$\epsilon$ & Uniaxial strain \\
\hline$\epsilon^{t}$ & Transformation strain tensor \\
\hline$\epsilon^{t-r}$ & Final transformation strain after forward transformation \\
\hline$\sigma$ & Uniaxial stress $(\mathrm{Pa})$ \\
\hline$\sigma^{\prime}$ & Deviatoric stress $(\mathrm{Pa})$ \\
\hline $\bar{\sigma}$ & Von Mises equivalent stress $(\mathrm{Pa})$ \\
\hline $\bar{\sigma}_{\text {crit }}$ & Critical von Mises equivalent stress $(\mathrm{Pa})$ \\
\hline$\Phi^{t}$ & Transformation function \\
\hline$\xi$ & Martensite volume fraction \\
\hline$\xi^{r}$ & Final martensite volume fraction after forward transformation \\
\hline$a_{1}, a_{2}, a_{3}$ & Transformation hardening coefficients \\
\hline$A_{f}$ & Austenite finish temperatur $\left({ }^{\circ} \mathrm{C}\right)$ \\
\hline$A_{s}$ & Austenite start temperature $\left({ }^{\circ} \mathrm{C}\right)$ \\
\hline$C^{A}$ & Austenite stress influence coefficient $\left(\mathrm{MPa} /{ }^{\circ} \mathrm{C}\right)$ \\
\hline$C^{M}$ & Martensite stress influence coefficient $\left(\mathrm{MPa} /{ }^{\circ} \mathrm{C}\right)$ \\
\hline$E_{A}$ & Austenite Young's modulus (GPa) \\
\hline$E_{M}$ & Martensite Young's modulus (GPa) \\
\hline$H^{c u r}$ & Maximum transformation strain at current stress \\
\hline$H_{\max }$ & Maximum attainable transformation strain \\
\hline$H_{\min }$ & Minimum transformation strain \\
\hline$k, k_{\text {exp }}$ & Exponential growth coefficient \\
\hline$M_{f}$ & Martensite finish temperature $\left({ }^{\circ} \mathrm{C}\right)$ \\
\hline$M_{s}$ & Martensite start temperature $\left({ }^{\circ} \mathrm{C}\right)$ \\
\hline$n_{1}, n_{2}, n_{3}, n_{4}$ & Transformation hardening exponents \\
\hline$S^{A}$ & Austenite compliance tensor \\
\hline$S^{M}$ & Martensite compliance tensor \\
\hline$T$ & Temperature $\left({ }^{\circ} \mathrm{C}\right)$ \\
\hline$T_{0}$ & Starting temperature $\left({ }^{\circ} \mathrm{C}\right)$ \\
\hline
\end{tabular}




\section{TABLE OF CONTENTS}

Page

ABSTRACT ...............................

ACKNOWLEDGEMENTS ............................ ii

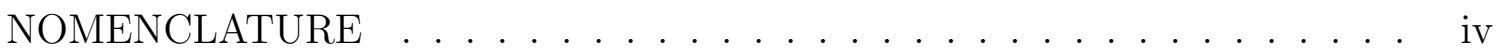

TABLE OF CONTENTS ...................... . . . . .

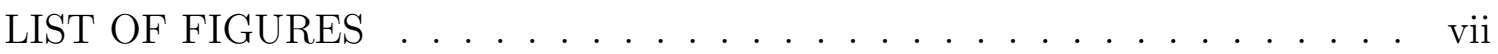

LIST OF TABLES ....................... . . . ix

1. INTRODUCTION AND LITERATURE REVIEW . . . . . . . . . . . . . 1

1.1 Literature Review: Shape Memory Alloys . . . . . . . . . . . . . . . . 1

1.1.1 Constitutive Modeling . . . . . . . . . . . . . 2

1.1.2 Material Parameter Characterization ........... . 3

1.2 Literature Review: Machine Learning . . . . . . . . . . . . . . . 7

1.2.1 Genetic Algorithms . . . . . . . . . . . . . . . 8

1.2.2 Reinforcement Learning . . . . . . . . . . . . . . . . . 9

1.2.3 Artificial Neural Networks . . . . . . . . . . . . . . . . . . . 9

1.3 Literature Review: Shape Memory Alloys and Machine Learning . . . 10

1.4 Approach Summary . . . . . . . . . . . . . . . 12

2. SHAPE MEMORY ALLOYS . . . . . . . . . . . . . . . . 16

2.1 Mechanics and Behavior . . . . . . . . . . . . . . . . 16

2.1.1 Austenite and Martensite Phases . . . . . . . . . . . . . . 16

2.1.2 One-way Shape Memory Effect . . . . . . . . . . . . . . . . 17

2.1.3 Pseudoelasticity . . . . . . . . . . . . . . . . . . 18

2.1.4 Two-way Shape Memory Effect . . . . . . . . . . . . . . . . 19

2.2 Targeted Material Parameter Selection . . . . . . . . . . . . . . 20

3. ARTIFICIAL NEURAL NETWORKS . . . . . . . . . . . . . . . 23

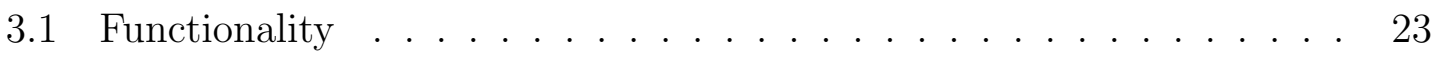

3.2 Structure . . . . . . . . . . . . . . . . . 26

3.3 Training . . . . . . . . . . . . . . . . . . . . 27

3.4 Design . . . . . . . . . . . . . . . . . . . . . . . . . . . . 28

4. TRAINING AN ANN FOR SMA CHARACTERIZATION . . . . . . . . . 30 
4.1 Model Implementation . . . . . . . . . . . . . . . . . . . . . 30

4.2 Training Data Generation . . . . . . . . . . . . . . . . . . . 33

4.2 .1 Factorial Method . . . . . . . . . . . . . . . . . . 35

4.2 .2 Taguchi Method . . . . . . . . . . . . . . 40

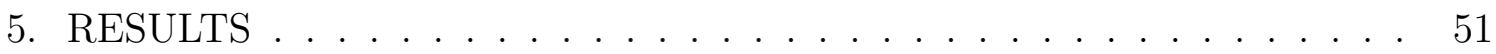

5.1 ANN Structure and Training . . . . . . . . . . . . . . . 52

5.2 One-Plot Method vs. Two-Plot Method . . . . . . . . . . . . . . 52

5.3 Factorial Approach . . . . . . . . . . . . . . . . . . . . 55

5.4 Taguchi-Factorial Approach . . . . . . . . . . . . . . 61

6. CONCLUSIONS AND RECOMMENDATIONS . . . . . . . . . . . . . 69

6.1 Conclusions . . . . . . . . . . . . . . . . . . . . . 69

6.2 Recommendations . . . . . . . . . . . . . . . . 70

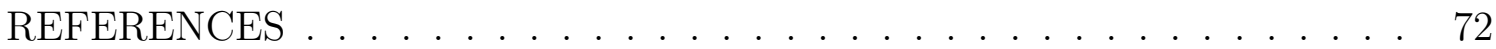




\section{LIST OF FIGURES}

1.1 Differential Scanning Calorimetry (DSC) method of SMA characteri-

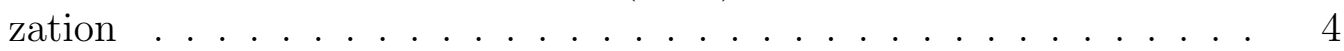

1.2 Example SMA hysteresis plot - strain-temperature coordinates . . . . 4

1.3 Example strain response method of SMA characterization - Data col-

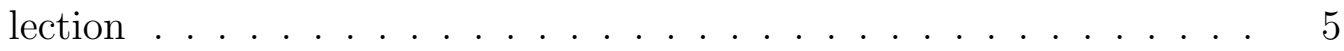

1.4 Example characteristic transformation temperatures identification . . 6

1.5 Example transformation strain behavior modeling . . . . . . . . . 6

1.6 SMA material parameter characterization using ANN process summary diagram . . . . . . . . . . . . . . . . . . . 14

2.1 Shape memory alloy transformation . . . . . . . . . . 17

2.2 Max transformation strain . . . . . . . . . . . . . . . . . . . 19

2.3 SMA training example $\ldots \ldots \ldots \ldots \ldots \ldots \ldots$

2.4 Characteristic SMA transformation temperatures (at zero stress) . . . 22

3.1 Typical ANN structure . . . . . . . . . . . . . . . . 25

3.2 A single artificial neuron . . . . . . . . . . . . . . . 25

3.3 Common activation functions $\ldots \ldots \ldots \ldots \ldots$

4.1 Model validations - literature example (top) versus implementation example (bottom) . . . . . . . . . . . . . . . . 34

4.2 Hysteresis coordinate selection for ANN input . . . . . . . . . . 35

4.3 Strain-temperature coordinates for a single training specimen . . . . . 40

4.4 Full training data set containing 360 specimens $\ldots \ldots \ldots$. . . . . 41

4.5 Full training data set containing 4296 specimens . . . . . . . . . . . 41

4.6 Baseline Taguchi-based training data set containing 26 specimens . . 46 
4.7 Full taguchi-based training data set containing 482 specimens . . . . 50

5.1 Study assessing ANN accuracy vs number of included hidden layer nodes . . . . . . . . . . . . . . . . . 53

5.2 Factorial method error comparison for different numbers of included training specimens . . . . . . . . . . . . . . . . 60

5.3 Taguchi-factorial nethod error comparison for different numbers of included training specimens . . . . . . . . . . . . . . . . . . . 64

5.4 Error histogram comparison of ANNs trained using random and Taguchibased methods of training data generation . . . . . . . . . . 66

5.5 Factorial vs. Taguchi-factorial method error comparison . . . . . . . . 67 


\section{LIST OF TABLES}

TABLE

Page

4.1 Training Data Parameter Values Bounds . . . . . . . . . . . 38

4.2 Training Data Parameter Values Using Factorial Method . . . . . . . 39

4.3 Taguchi Method Application: Example $1 \ldots \ldots \ldots$

4.4 Taguchi Method Application: Example $2 \ldots \ldots \ldots$

4.5 Taguchi Method Application: Example $3 \ldots \ldots$. . . . . . . . 44

4.6 Baseline Training Data Parameter Values using Taguchi-based Method 46

4.7 Training Data Parameter Values using Taguchi-based Method . . . . 47

4.8 L50 Orthogonal Array . . . . . . . . . . . . . . . . . . . . . . 49

5.1 Example SMA Training Specimen Parameters . . . . . . . . . . . 54

5.2 Comparison of 1-Plot and 2-Plot Methods . . . . . . . . . 55

5.3 Batch Analysis - Target Values _. . . . . . . . . . . . . 56

5.4 Batch Analysis - Factorial Method (ANN Output Values) . . . . . . 57

5.5 Batch Analysis - Factorial Method (Error Values) . . . . . . . . 58

5.6 ANN Performance Comparison: Factorial Approach . . . . . . . . . . 59

5.7 Batch Analysis - Taguchi-Factorial Method (ANN Output Values) . . 62

5.8 Batch Analysis - Taguchi-Factorial Method (Error Values) . . . . . 63

5.9 ANN Performance Comparison: Taguchi-Factorial Approach . . . . . 63

5.10 ANN Performance Comparison: Random vs Taguchi-Factorial Methods 65

5.11 ANN Performance Comparison: Taguchi vs. Factorial . . . . . . . . 68 


\section{INTRODUCTION AND LITERATURE REVIEW}

This thesis develops and assesses a novel method of shape memory alloy (SMA) material parameter identification. The intent here is not to replace existing SMA constitutive models, nor is it to fundamentally change existing characterization methods. Rather, the intent is to facilitate and supplement the existing SMA material parameter characterization process, allowing more rapid characterization of SMAs while requiring less data and SMA expertise. This thesis works towards this goal by applying a computational intelligence method to the task of SMA material parameter characterization. Specifically, this thesis develops a means of simplifying the SMA characterization procedure by using a machine learning method - an artificial neural network.

\subsection{Literature Review: Shape Memory Alloys}

Shape memory alloys have demonstrated unique utilization in a variety of contexts in recent years, spanning such diverse fields as medicine, robotics, and aerospace structures. In the medicinal field, SMAs have been used in the manufacturing of stents - small mesh tubes that are typically inserted into narrow or weak arteries to improve bloodflow [3]. The use of SMAs in this context improves overall stent performance by facilitating the insertion and placement of stents in arteries. With proper selection of material, the unique properties of SMAs allow for a stent that is able to "morph" between two geometric configurations: a small diameter configuration for use during insertion and placement, and a larger diameter configuration that widens the artery once the stent is in place. In the field of robotics, SMAs have been used to achieve a variety of functions. As a single example, SMAs have been used in develop- 
ing an earthworm-like micro robot [19]. In this application, SMAs formed the basis of a two-way actuator that connects front and rear body modules of the robot. A small battery carried on-board causes the SMA actuator to expand and contract, slowly propelling the robot forward. As a final example, SMAs have been implemented in the design of a variable-geometry jet engine chevron [12]. In this implementation, SMA-based actuators allow realization of active engine flaps that exhibit maximum deflection at takeoff and landing - decreasing jet engine noise - while avoiding flow deflection during normal flight.

\subsubsection{Constitutive Modeling}

Due to the far-reaching utility exhibited by SMAs, much work has focused on the development of SMA constitutive models and their numerical implementations. Models developed by Liang and Rogers [27], Boyd and Lagoudas [2], and Hartl [11] have been proven to provide accurate mathematical description of several aspects of SMA behavior. Liang and Rogers [27] present a multi-dimensional thermomechanical SMA model based on a combination of micromechanics and macromechanics in which the model is governed by an introduced martensite fraction variable and subsequently used to solve the torsion problem of an SMA rod. Boyd and Lagoudas [2] model pseudoelasticity and the shape memory effect using a free energy function and dissipation potential, exploring three differing cases based on selected internal state variables. Hartl [11] develops and experimentally validates three phenomenological SMA models based on continuum thermodynamics: one for fully recoverable SMA response that includes modeling of material hardening during transformation and transformation strain dependence on applied stress, one for rate-independent plastic deformations, and one for rate-dependent viscoplastic deformations. This model in particular, termed the Hartl-Lagoudas model in this thesis, is discussed further in 
Section 4.1.

\subsubsection{Material Parameter Characterization}

Though largely capable of accurately modeling SMA behavior, the constitutive models documented in the literature inherently depend on the accurate characterization of the material - typically in the form of identifying values for material parameters. Developing a means of consistently and accurately identifying these unique SMA physical parameters is not a novel task; different methodologies have been studied. One such method is Differential Scanning Calorimetry (DSC). DSC is a technique in which a specimen is held at a constant stress and constant rate of temperature change, and energy required to maintain this rate of temperature change is recorded [33]. Using this method, illustrated in Fig. 1.1, one can identify the region of phase transformation and determine the related stress-specific transformation temperature material parameters [25].

Another method often used to identify transformation temperatures involves analyzing hysteretic SMA strain response caused by either changes in temperature under constant applied stress, or changes in applied stress while kept at constant temperature [4]. Due to its prevalence, the former method here (constant applied stress with varying temperature) will be the focus of SMA characterization discussion. Because SMA strain response exhibits hysteresis (see Figure 1.2) that varies with applied stress, accurate identification of characteristic SMA material parameters necessitates extensive data collection. First, strain response data must be collected for a single specimen at several different cases of constant applied stress (Figure 1.3) [12]. Once this data is collected, stress-specific transformation temperatures must be identified. A more detailed discussion of these transformation temperatures is found in Chapter 2. These stress-specific parameters must then be used to identify 


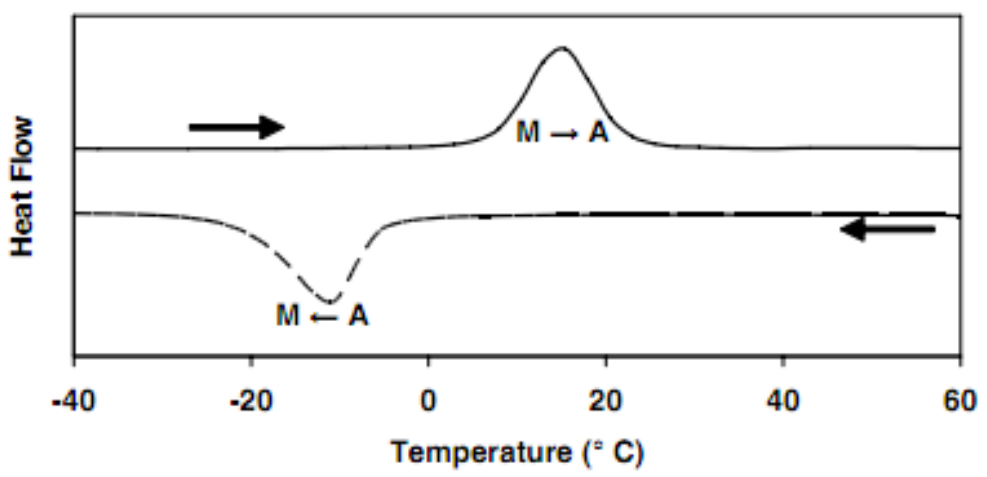

(a) DSC results

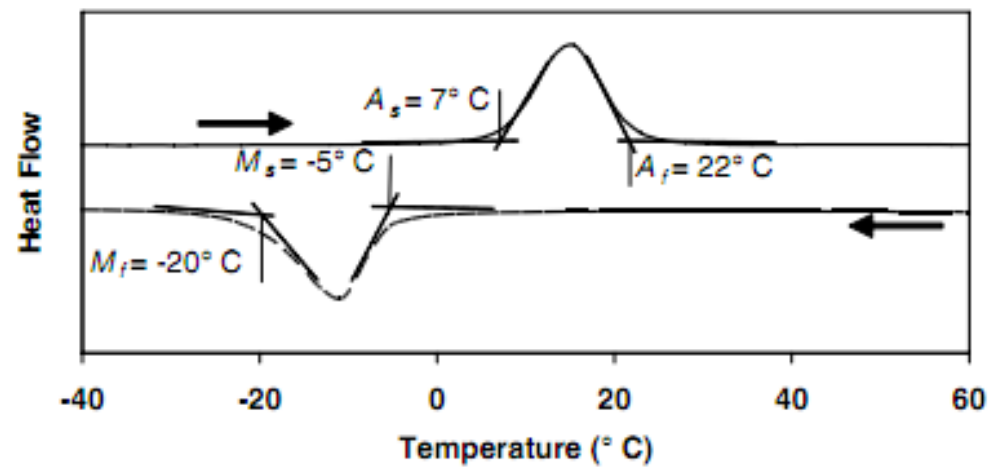

Figure 1.1: Differential Scanning Calorimetry (DSC) method of SMA characterization

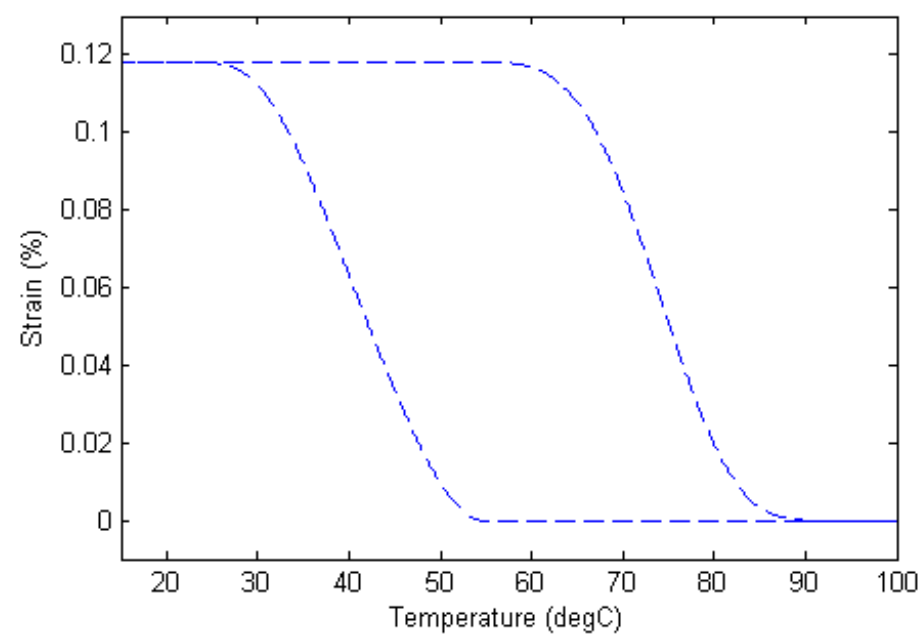

Figure 1.2: Example SMA hysteresis plot - strain-temperature coordinates 


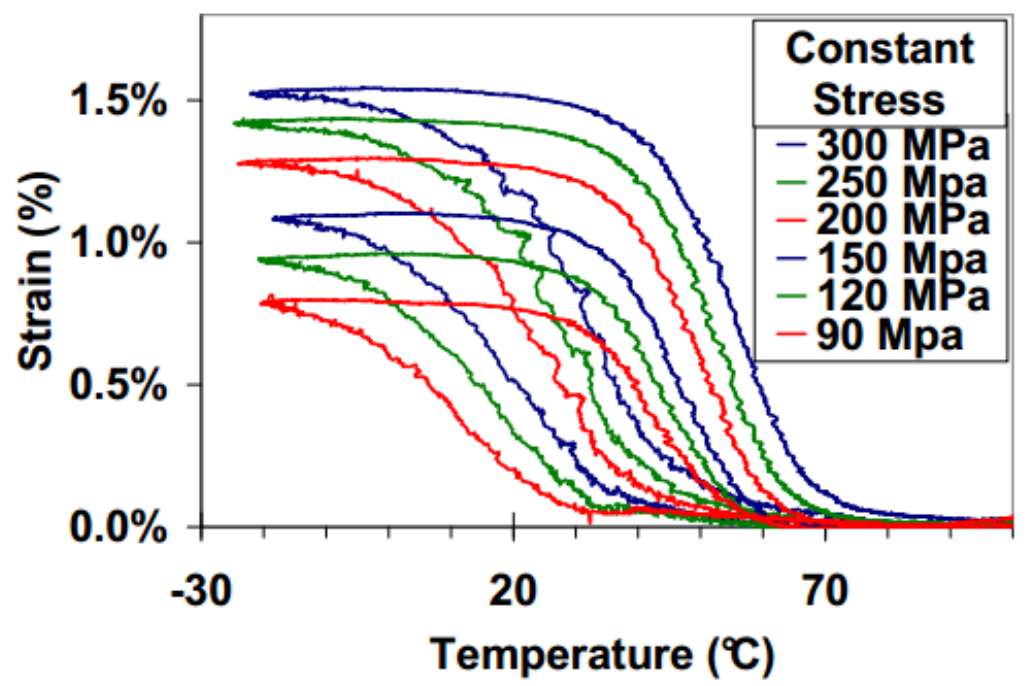

Figure 1.3: Example strain response method of SMA characterization - Data collection

the characteristic, stress-invariant transformation temperatures of the material (Figure 1.4) [12]. Depending on the model used, additional data processing is required to obtain the parameters governing maximum transformation strain variation with applied stress (Figure 1.5) [12]. This strain response-based method of SMA material parameter identification is often advantageous to the DSC method in that its required equipment for data collection is more widely available and less specialized.

As evidenced above, although these methods allow one to identify SMA material properties with some degree of accuracy, these existing methods are currently time-consuming procedures requiring both extensive data collection and processing. Furthermore, these processes typically require some degree of SMA expertise. This creates a somewhat high barrier of entry for both engineers hoping to use the material and researchers desiring to further model advanced behavior of the material. 


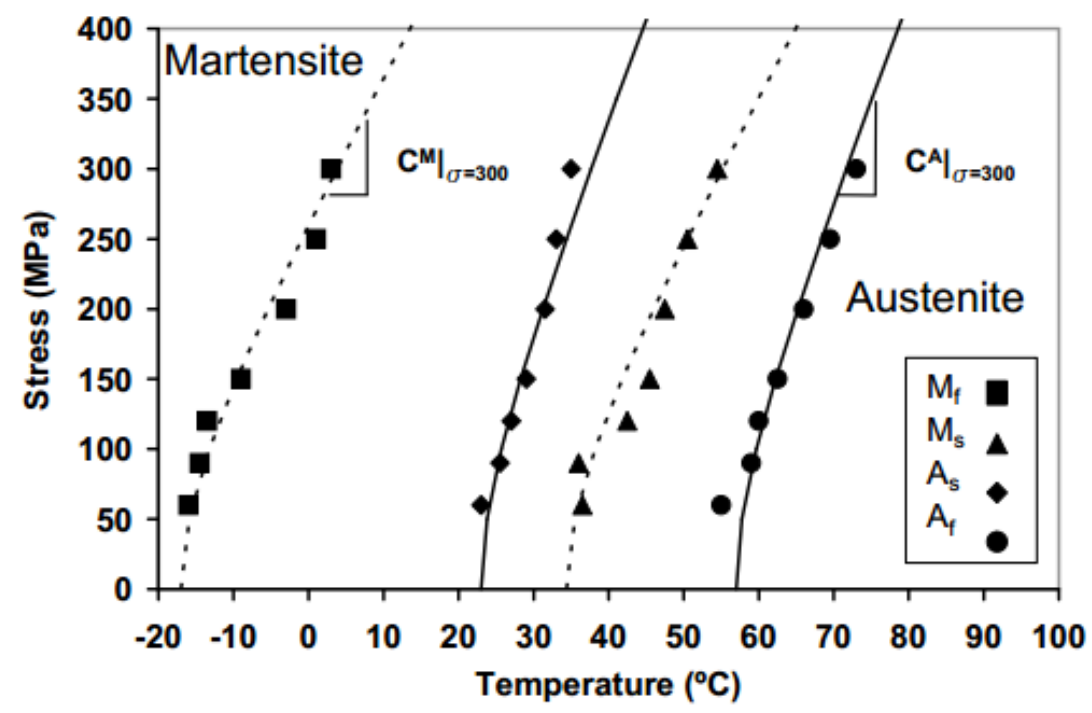

Figure 1.4: Example characteristic transformation temperatures identification

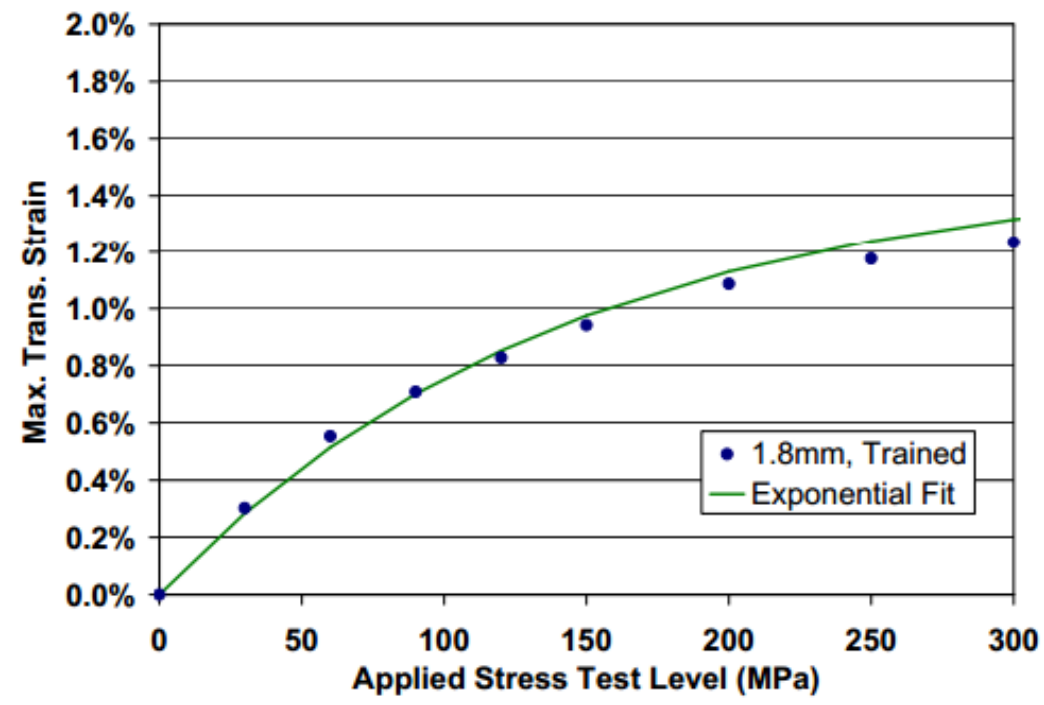

Figure 1.5: Example transformation strain behavior modeling 


\subsection{Literature Review: Machine Learning}

Machine learning - the development of computational algorithms through which a computer adapts its behavior based on given data - is not a novel topic, originating in the literature (at least on a theoretical level) over a half-century ago. Rudimentary methods of machine learning were proposed and studied as early as the 1950s - a 1951 publication by Walter [37] presented a simple artificial nervous system that could associate two stimuli with corresponding effects through a "learning" box. The developed "learning box" in this case consisted of an analog circuit that could perform the various simple operations necessary to develop the desired association. By learning positive and negative associations with light and touch, a simple machine was taught how to avoid obstacles in moving towards a light source. Another example of early machine learning theory is found in a 1958 publication by Friedberg [7]. In this work, Friedberg describes a general computational learning method that intends to teach a machine how to perform a given task. The method seeks to accomplish this, however, not by specifying specifics rules. Rather, it does this by allowing the machine to try procedures either at random or according to an otherwise unintelligent system and then periodically informing the machine whether or not it is performing as desired.

Although machine learning was initially limited to relatively simple tasks due to the generally large amount of required computational time, it has risen in prominence in recent years with the rising affordability of high-speed computation. Machine learning today has seen numerous practical uses and, to name one prominent example, is often used in the context of pattern recognition. The literature contains a wealth of information dedicated to the general topic of machine learning pattern recognition - one example is provided by Bishop [1]. In this text, Bishop provides a 
thorough and detailed overview of both traditional and machine learning-based pattern recognition methods. Developments and example implementations are provided for topics spanning from linear models for regression and classification to neural networks.

Specific developments regarding machine learning in the past few decades have included the introduction of topics such as genetic algorithms, reinforcement learning, and artificial neural networks.

\subsubsection{Genetic Algorithms}

A genetic algorithm (GA) is a heuristic search technique often used in optimization tasks that draws inspiration from the process of natural selection. Generally seeking to optimize a specified performance metric, a GA explores and assesses possible solutions through mutation. That is, the GA will typically be initialized with a number of proposed solutions. These solutions will then be ranked according to a specified performance metric. The solutions exhibiting the highest performance will form the basis of the next generation of possible solutions, and low performing solutions will be discarded. A variety of small changes - mutations - will be made to the passed on high performing solutions so as to ensure variety in the subsequent generation. This process of mutation, assessment, and elimination is repeated until a specified criteria is met, such as a desired performance value.

Genetic algorithms excel in applications in which a performance metric is easily defined, and in which the explored solution domain can easily take on a binary representation.More detailed information is readily available in the literature, with comprehensive overviews and developments from Goldberg [9] and Davis [5] as two often-cited examples. 


\subsubsection{Reinforcement Learning}

Reinforcement learning (RL) is a form of machine learning that draws inspiration from behavioral psychology. RL implementations are focused around a software agent that seeks to maximize reward through actions performed in a domain of environment states. The agent is generally given a set of discrete possible actions, and two primary sets of rules are put in place: one that governs how the agent transitions between environment states, and one that determines a scalar reward for each state transition. As the agent moves through discrete time steps, selecting possible actions at the environment states it encounters, it stores reward values for each discovered stateaction pair. The agent is able to "remember" these rewards at each environment state, and as it seeks to maximize total accumulated reward, the agent both exploits the knowledge of these known reward values and explores unknown state-action pairs. Over time, a successful agent will explore the environment and identify a "path" that maximizes performance according to the reward rules.

RL is well suited to tasks in which environment domains are known and easily discretized. Furthermore, because an RL agent generally seeks a desirable action sequence based on a performance metric, it is often well suited to controls-based tasks. Sutton and Barto [35] and Kaelbling, Littman, and Moore [17] provide thorough overviews of RL methods that are widely referenced in the literature.

\subsubsection{Artificial Neural Networks}

Artificial neural networks (ANNs) represent a third form of machine learning. Just as genetic algorithms and reinforcement learning draw inspiration from natureGAs in the form of natural selection, and RL in the form of behavioral psychologyANNs draw inspiration from biological central nervous systems, such as the brain. Mimicking their natural counterparts, ANNs take the form of networks consisting 
of a number of interconnected nodes. Connections between the nodes, or neurons, allow mapping of information between input and output pairs given to the ANN. The process in which an ANN "learns" this mapping is called training. In training, a number of input values with known corresponding output values are given to the ANN. Based on this supplied training data, connection weights are adjusted throughout the network until the output given by the ANN matches the desired outputs for various input values. Because this training involves data in which target values are known, this is referred to as supervised learning. Once trained, the ANN is capable of "predicting" output values for supplied inputs that are not contained within the training data. Functionally speaking, the ANN learns the pattern and makes predictions based on it.

ANNs excel, as mentioned, in the context of pattern recognition. Specifically, they are best suited to pattern recognition problems in which correct input-target training data is prevalent. ANNs are discussed in somewhat greater detail in Chapter 3. Further description of ANNs is widely available in the literature - two examples being the publications of Hagan, Demuth, and Beale [10] and Mehrotra, Mohan, and Ranka [29].

\subsection{Literature Review: Shape Memory Alloys and Machine Learning}

Although machine learning methods have been occasionally applied to specific context of SMAs in the past, these studies have largely focused on hysteresis com-

pensation in active feedback control systems. For example, Song, Chaudhry, and Batur [34] present a method of SMA actuator control tracking using, in part, ANNs. The experimental test set-up utilizes a single nickel titanium (NiTi) SMA wire and a bias spring. Training data is generated with a sinusoidal voltage signal applied to 
the SMA wire, and the generated hystereis data is applied towards the implementation of a neural network that governs hysteresis compensation. The utilized control system consists of a proportional-derivative (PD) controller working in tandem with a nonlinear sliding-mode controller. This control system yields a satisfactory level of tracking across a select number of tested frequencies.

In [28], Ma, Song, and Lee aim to control a NiTi SMA wire actuator using the changing internal electrical resistance of the metal as the state variable. After describing the experimental setup (a uniaxially loaded wire with tension spring, a position sensor, and driving deformation with applied electrical current), some obtained experimental data are presented relating electrical resistance and voltage to displacement. Unsteady hysteresis in the electrical resistance-displacement relationship is identified, motivating the implementation of machine learning. An ANN is implemented to relate electrical resistance to displacement, and this is used with a PD controller for precise actuation. This is concluded to be a feasible alternative to position sensor-based control methods.

The theme of SMA hysteresis compensation using machine learning methods is continued in [18], in which Kilicarslan, Song, and Grigoriadis aim to develop a SMA actuator controller specifically compensating for hysteresis by means of an adaptive neuro fuzzy inference system (ANFIS) and PI controller. The experimental setup is described - involving a thin SMA wire, a bias spring, a laser displacement sensor, and electrodes from SMA actuation - and the predictions from the resulting ANFIS are presented. By reversing the inputs and outputs of the ANFIS, a controller is developed and tested with step inputs. In order to fully compensate for the voltagedisplacement hysteresis, a proportional-integral (PI) controller is included. With this, given a step input, a settling time zero steady-state error are achieved that are concluded to be satisfactory for general purposes. 
Kirkpatrick and Valasek [21, 22, 23] have similarly explored SMA active length control and hysteresis characterization using a reinforcement learning approach. This work largely focuses on using reinforcement learning to map a relationship directly between voltage applied across a thin wire SMA specimen and corresponding SMA strain with a goal of achieving precise active SMA length control. The approach is deemed to be more effective than previous reinforcement learning approaches due to the fact that it does not necessitate development of a relationship between temperature and applied voltage; furthermore, this voltage-strain approach is shown to be more accurate than a temperature-strain approach because measurements of applied voltage can be taken more accurately than measurements of the SMA wire temperature.

\subsection{Approach Summary}

Rather than focusing on SMA hysteresis compensation for control purposes, this thesis develops an approach in which a machine learning technique is used to facilitate the SMA characterization process. More specifically, this thesis develops a method in which an ANN is trained to identify a number of SMA-specific material parameters (transformation temperatures and stress influence coefficients) given strain-temperature hysteresis data. Training data is generated using an existing constitutive SMA model, which allows rapid generation of input-target pairs for ANN training-ANN input values being strain-temperature coordinates, and ANN target values being the desired SMA material parameters. Parameter selection for the training data is performed using two different methods - factorial and Taguchieach using the same specified minimum and maximum parameter value ranges. The performance of each trained ANN is evaluated by generating hysteresis data for 
a number of random evaluation SMA specimens, applying them as inputs to the ANN, and comparing the ANN output values with the known target evaluation SMA specimen parameter values. The number of generated training specimens is iteratively updated until a satisfactory ANN accuracy value is achieved for each of the implemented methods of training data generation. This process is summarized in Figure 1.6.

The decision to approach the task of SMA material parameter identification with an ANN was driven by the pattern recognizing reputation of ANNs. The task of identifying SMA material parameters based on strain-temperature can be intuitively imagined as a pattern recognition problem. A specific combination of SMA material parameters will correspond to a specific "shape" of the material's strain-temperature hysteresis plot. If one studies enough examples of these hysteresis plots and has knowledge of their corresponding material parameters, it intuitively makes sense that a pattern could be identified, allowing identification of the material parameters.

Other possible machine learning techniques were eliminated as viable options for a variety of reasons. For example, because SMA material parameters span a range of possible values, the solution space to this problem is not easily made to take the form of a binary representation - this eliminated the prospect of using a genetic algorithm for parameter identification. An ANN was selected over an implementation of reinforcement learning primarily due to the fact that a large amount of inputtarget data for SMA characterization is relatively easily accessible through the use of existing constitutive models - given SMA material parameters, an SMA constitutive model outputs strain-temperature hysteresis data. Furthermore, the characterization problem is not intuitively posed as a software agent-based state exploration problem.

This paper is organized as follows. After concluding the relevant introductory material in Chapter 1, further detailed background information is provided for both 


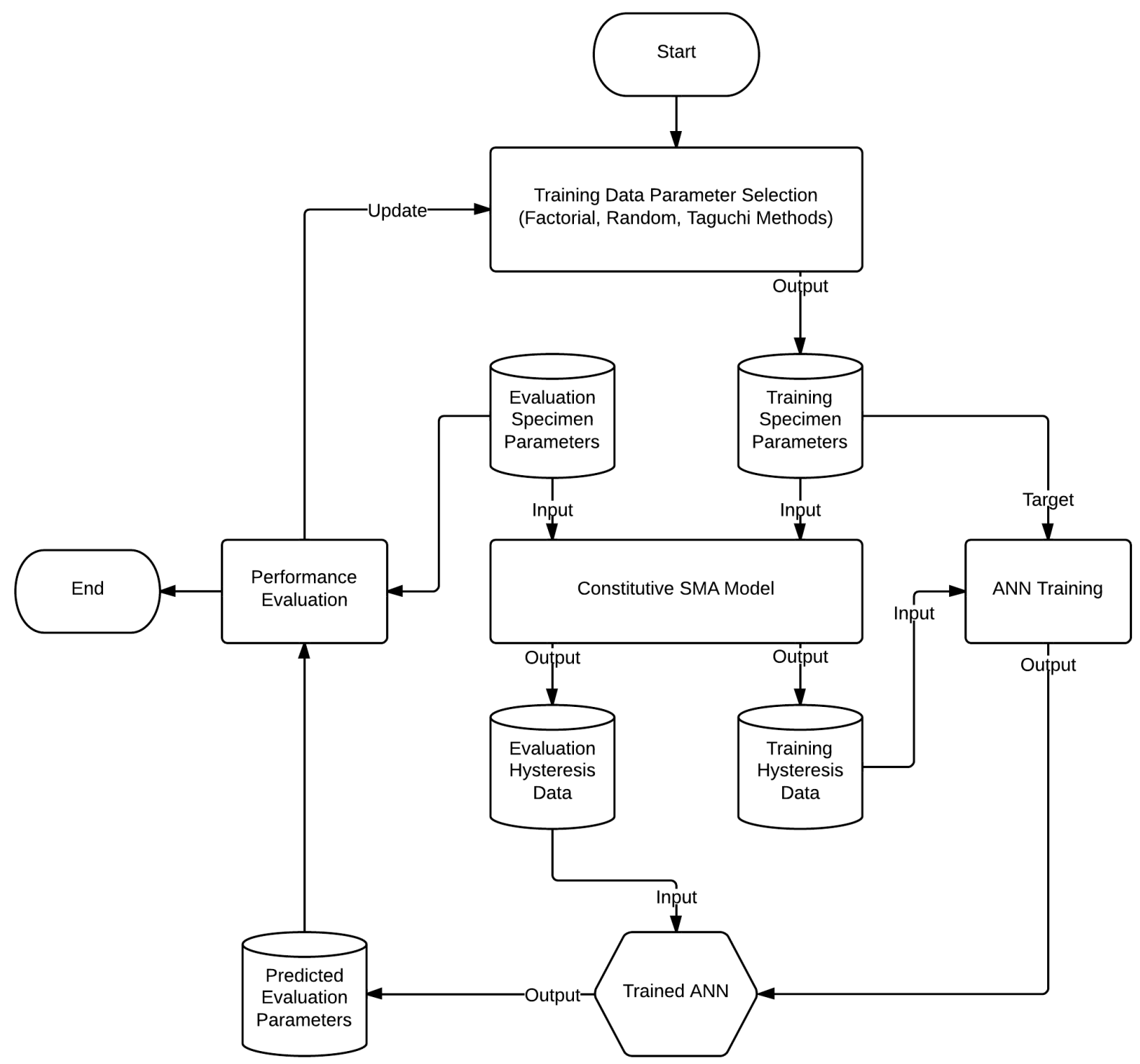

Figure 1.6: SMA material parameter characterization using ANN process summary diagram 
SMAs and ANNs in Chapters 2 and 3, respectively. The process of training an ANN for SMA characterization is then discussed at some length in Chapter 4, subdivided into sections discussing the constitutive model implemented to generate training data in Section 4.1 and the methods used to generate said training data in Section 4.2. Results are then presented in Chapter 5, comparing the different methodologies utilized. Finally, conclusions and recommendations are given in Chapter 6 . 


\section{SHAPE MEMORY ALLOYS}

With an end goal of identifying SMA material properties, it is first necessary to understand the unique mechanics and behavior of SMAs. Once these mechanics and their corresponding properties are understood, it is then necessary to select the material properties that will be targeted for characterization.

\subsection{Mechanics and Behavior}

The literature contains a wealth of references focused solely on the topic of SMA mechanics and behavior, ranging from experimentally and empirically-derived discussions of nuanced SMA behavior to theoretical derivations of the underlying mechanics based on fundamental laws of physics. Such references are readily available to anyone who seeks a more in-depth knowledge of SMAs - one such example provided by Lagoudas [25]. A complete discussion of SMAs, however, is beyond the scope of this work. With the stated goal of characterizing material properties, the focus of this section is to introduce the different material properties that govern SMA behavior.

\subsubsection{Austenite and Martensite Phases}

The unique characteristics of an SMA are the result of a solid-to-solid phase transformation between two crystalline structures. While in the higher-temperature austenite phase, the SMA will typically exhibit a cubic crystalline structure. If the material is cooled to below the characteristic martensitic start temperature $\left(M_{s}\right)$, it will undergo a forward transformation into its lower-temperature martensite phase. The martensite phase, characterized by a tetragonal, orthorhombic, or monoclinic crystalline structure is fully reached once the temperature is equal to or less than the 


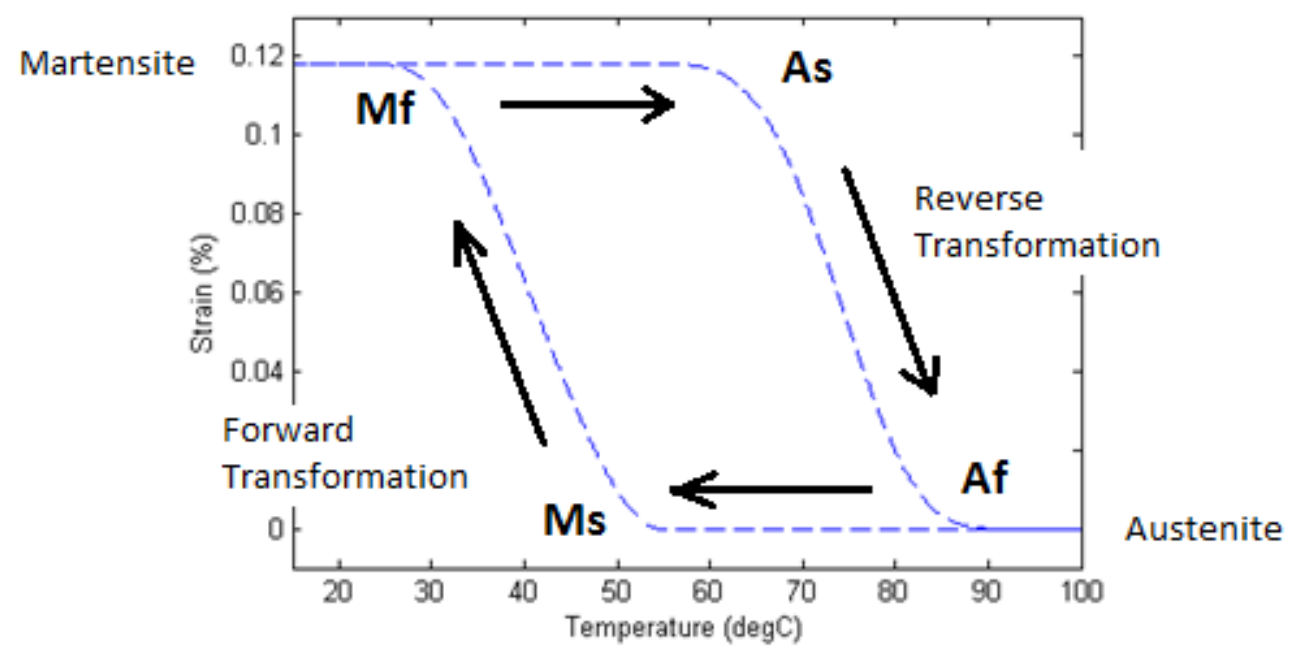

Figure 2.1: Shape memory alloy transformation

martensitic finish temperature $\left(M_{f}\right)$. This process is reversible - if the martensitic structure is heated to a temperature greater than the austenitic start temperature $\left(A_{s}\right)$, the SMA will undergo a reverse transformation, returning to pure austenite after reaching the austenitic finish temperature $\left(A_{f}\right)$. Although phase transformation can be achieved by simultaneous variation of both applied stress and temperature, experimentation typically involves constraining one parameter (temperature or stress) at a fixed value and varying the other. An illustration of this phase transformation, using fixed stress, is shown in Figure 2.1.

\subsubsection{One-way Shape Memory Effect}

If no stress is applied to the SMA during the forward transformation, the end result is twinned martensite, in which there is no dominant martensitic crystal orientation. In this case, the change in crystalline structure is self-accommodating, causing there to be no change in macroscopic geometry. If returned to fully austenite, there would consequently be no transformation strain generation. While the SMA is in a 
twinned martensite phase, however, one can create a dominant crystal orientation, however, by applying stress greater than or equal to the detwinning starting stress. This creates detwinned martensite and results in a macroscopic "plastic" change in geometry of the SMA. The unique shape memory effect (SME) of an SMA is observed if the detwinning process is conducted at a temperature lower than $A_{s}$, and the resulting detwinned martensite is then heated to a temperature greater than $A_{f}$. In such a scenario, the SMA will transform back to fully austenite and return to its original non-deformed geometry - that is, the seemingly plastic strain will be recovered. This phenomenon is termed the one-way shape memory effect (though often referred to as simple the shape memory effect), because in this case shape recovery only occurs with an increase in temperature after mechanically-induced detwinning. The strain recovery in this case would generate a transformation strain with a magnitude equal to the that of the initial inelastic strain. The maximum magnitude of this transformation strain for a given SMA specimen varies with applied stress. This relationship necessitates additional material properties for constitutive modeling. As an example, one such model developed by Dr. Darren Hartl and Dr. Dimitris Lagoudas at Texas A\&M University [11] applies a curve fit of the form

$$
H_{\text {cur }}(\bar{\sigma})=H_{\text {min }}+\left(H_{\text {max }}-H_{\text {min }}\right)\left(1-e^{-k\left(\bar{\sigma}-\bar{\sigma}_{\text {crit }}\right)}\right)
$$

to a series of transformation strain values taken at different applied stresses. An example curve fit is shown in Figure 2.2 [11].

\subsubsection{Pseudoelasticity}

The fact that transformation strain varies with applied stress significantly impacts the modeling of the aforementioned transformation temperatures. An important characteristic of SMAs, consequently, is that the described transformation tempera- 


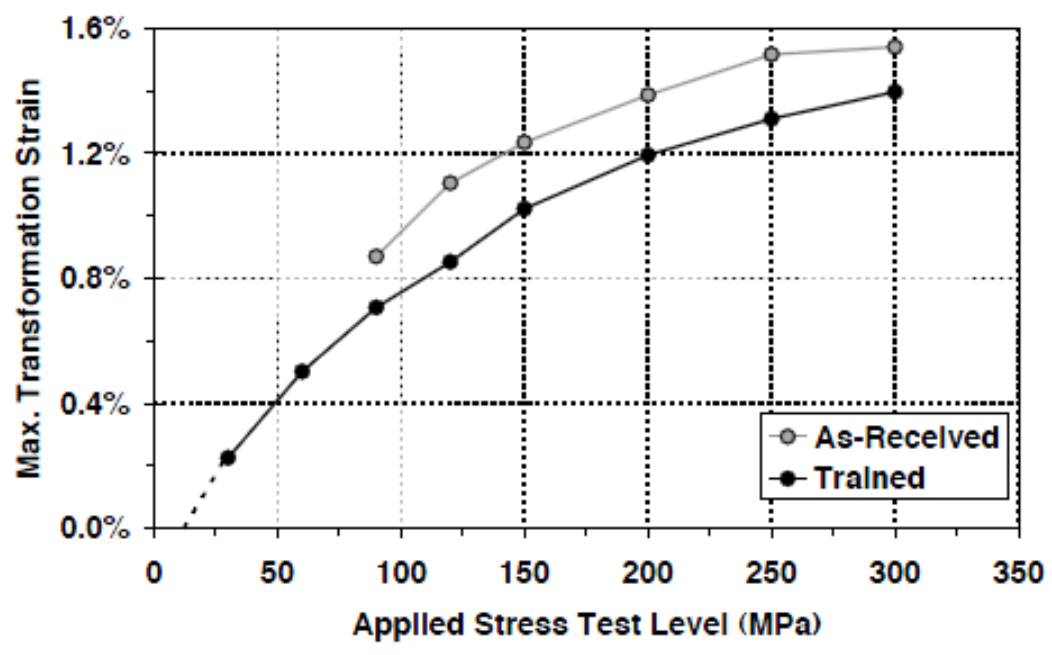

Figure 2.2: Max transformation strain

tures strongly depend on applied stress - each transformation temperature increases with the magnitude of applied stress. Transformation from austenite to detwinned martensite can therefore be accomplished by applying a high enough stress (at least greater than the detwinning starting stress) such that $M_{f}$ becomes greater than the ambient temperature. The SMA will consequently transform from austenite directly to detwinned martensite in one continuous process. If the ambient temperature is greater than the unloaded $A_{f}$, the material will immediately revert to austenite upon removal of the applied stress. This behavior is macroscopically observed as deformation and a subsequent return to the initial shape. This phenomenon is called pseudoelasticity.

\subsubsection{Two-way Shape Memory Effect}

In addition to the one-way shape memory effect and pseudoelasticity, there is also a behavior termed two-way shape memory effect (TWSME). To achieve the TWSME, an SMA must first undergo training, cyclic thermo-mechanical loading 


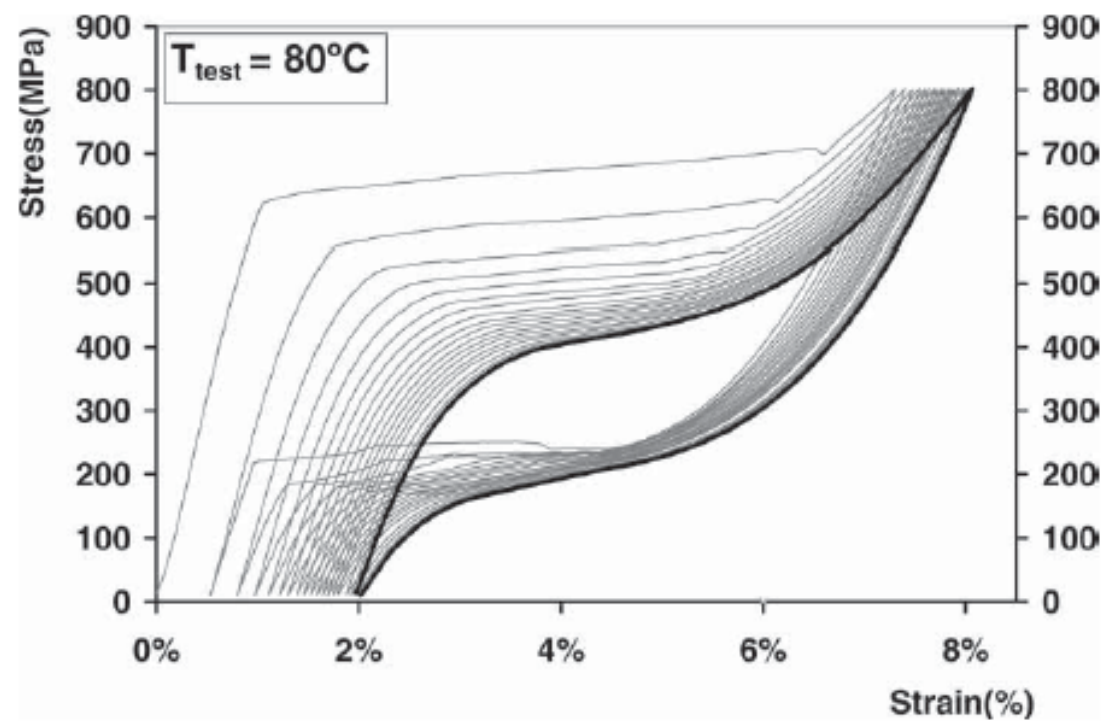

Figure 2.3: SMA training example

between two fixed states. Training is typically conducted until the hysteretic behavior of the material stabilizes - an example of which is shown in Fig. 2.3 [14]. As the SMA undergoes repeated loading cycles, a finite value of non-recoverable plastic strain is created. After some number of loading cycles, the growth of this plastic strain becomes negligible. Once trained, an SMA can exhibit TWSME, observed as a repeatable macroscopic change in shape caused by a purely thermal cyclic load.

\subsection{Targeted Material Parameter Selection}

Having introduced a number of SMA-specific material parameters, it is now necessary to select that parameters that will targeted for identification in this thesis. This selection is driven in part by the relative influences of the different parameters on thermomechanical behavior, and largely by the relative effort required for characterization of the different parameters using existing methods. To illustrate, 
a parameter that significantly impacts SMA behavior and that is difficult to numerically identify using existing characterization methods is a more desirable target than a parameter that is relatively easy to identify and that does not as significantly influence SMA strain response.

Transformation temperatures $\left(M_{s}, M_{f}, A_{s}, A_{f}\right)$ are perhaps the most definitive physical parameters of a given SMA. These temperatures govern the transformation between the two phases of the SMA and are unique to the material. As previously mentioned, values can be obtained for these parameters by observing strain generation within an SMA specimen as it undergoes changes in temperature while under constant stress. This method delimits the phase transformation region by evaluating the hysteretic region between austenite and martensite shown in Fig. 2.1. Stressspecific transformation temperatures can be found by noting changes in the strain rate of change with respect to temperature. The characteristic transformation temperatures of the SMA, independent of applied stress, can subsequently be found by plotting the changes in stress-specific transformation temperatures with respect to applied stress, as seen in Figure 2.4 [25]. The stress-specific transformation temperatures are shown with the solid shapes.

It is important to remember that transformation temperatures are a function of applied stress. Because of this, a given SMA has stress-specific transformation temperatures that are not to be confused with the final stress-invariant transformation temperatures of the material-indicated, for example, in Figure 2.4 where stress equals zero. These stress-invariant transformation temperatures will be four of the parameters targeted for characterization in this research. It is, however, furthermore desirable to model how the stress-dependent transformation temperatures vary with applied stress. This behavior is modeled with the stress influence coefficients $\left(C^{M}\right.$ and $\left.C^{A}\right)$, and it is illustrated in Figure 2.4 with approximated slope values 


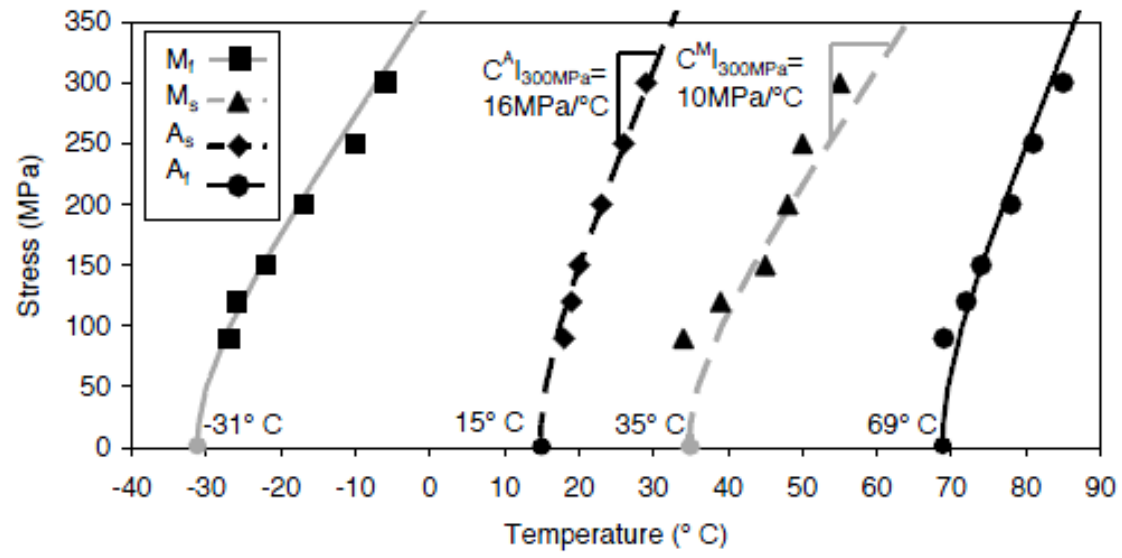

Figure 2.4: Characteristic SMA transformation temperatures (at zero stress)

[11]. These stress influence coefficients will be two additional parameters targeted for characterization. Six material parameters in total have therefore been selected for characterization: the austenite start and finish transformation temperatures $\left(A_{s}\right.$, $\left.A_{f}\right)$, the martensite start and finish transformation temperatures $\left(M_{s}, M_{f}\right)$, and the austenite and martensite stress influence coefficients $\left(C^{A}\right.$ and $\left.C^{M}\right)$. 


\section{ARTIFICIAL NEURAL NETWORKS}

As with SMAs, a comprehensive discussion of ANNs is beyond the scope of this work. The literature is rich with sources focused entirely on providing detailed information about ANNs, and such sources are readily available to those interested in learning more about the functionality of ANNs beyond what is explained in this thesis. Specific examples are the works of Hagan [10] and Mehotra [29]. The intent of this chapter is to provide enough information so as to convey an understanding of ANNs that supplements and facilitates explanation of the developed SMA material parameter characterization method, rather than detracting from it.

\subsection{Functionality}

As introduced in Section 1.2.3, an ANN is a machine learning implementation inspired by biological neural networks. Natural neural networks consist of a complex three-dimensional network of interconnected neurons passing information between one another in the form of synapses. These neurons, when considered individually, are severely limited in their computational capabilities. When considering the entirety of the network, however, these constructs are capable of staggeringly complex functionality, allowing such nuanced behavior as human cognition. Artificial neural networks attempt to mimic this behavior in a simplified sense, seeking to perform complex tasks through a system of individually simple components.

ANNs are typically tasked with identifying relationships between given inputs and targets and have repeatedly performed well in this context. For example, Rowly [31] demonstrated successful application of an ANN in the context of human face detection. Supplied with a number of example face and nonface images, several col- 
laborating ANNs were trained to detect the presence and locations of faces in images involving a wide variety of lighting, pose, facial expression, and identity conditions. To perform this task, an ANN first estimated orientations for any potential faces contained in the image. This ANN then passed the potential face and estimated orientation information to ANNs specialized in detecting frontal, half profile, and full profile face images. Another example application of an ANN is presented by LeCun [26], in which an ANN is used for recognizing handwritten numerals. Using several thousand handwritten US zip code numerals provided by the U.S. Postal Service, a single ANN was successfully trained to correctly recognize the handwritten numerals when given a 256 pixel digitization of each handwritten number. A third example of ANN pattern recognition is given by Kirkpatrick, May, and Valasek [20]. In this implementation, an ANN was trained to assist in the aircraft system identification process. Latitudinal-directional and longitudinal flight data was given to an ANN seeking to map a relationship between states and controls between time-steps. The trained ANN was shown to be capable of identifying A and B linear system matrices that matched the modeling accuracy of the existing Observer/Kalman Filter Identification method.

The pattern recognizing functionality exhibited by ANNs is accomplished by modifying the strengths of the connections between the individual simulated neurons, or nodes. The goal of this training process is to yield a non-parametric, "black box" model of the system based on the supplied training data. That is, a trained ANN will map a desired pattern between inputs and outputs, but it will not provide rigorous insight into the inner workings of the relationships between the input and output. 


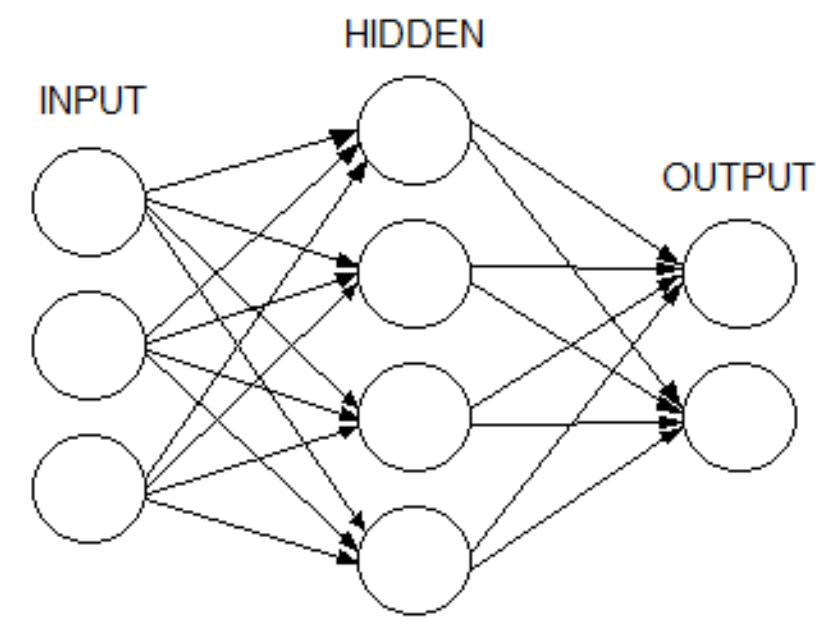

Figure 3.1: Typical ANN structure

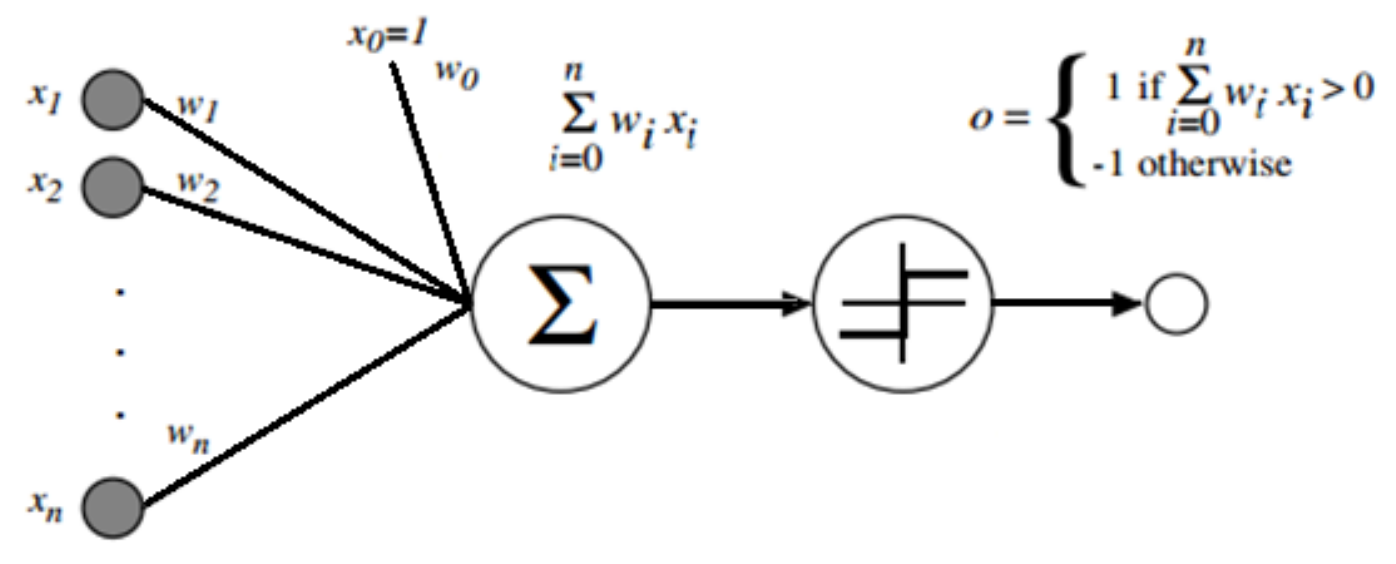

Figure 3.2: A single artificial neuron 


\subsection{Structure}

A typical ANN consists of several layers of simulated neurons - an input layer, an output layer, and typically at least one intermediate hidden layer-each node sending information along connections to the neurons of nearby layers. An example ANN structure with a single hidden layer is shown in Fig. 3.1. Note that this specific illustration demonstrates a feed-forward network, in which information is passed in one direction across the input, hidden, and output layers. Other ANN node organization methods do exist, but this feed-forward structure is the most prevalent and will be the focus of this section. In general, each neuron is a mathematical function that calculates the sum of inputs from nearby neurons and, optionally, a bias input. This summation is calculated taking into account the current strength, or weight, of each neuron-to-neuron connection. The resulting value is then passed through a transfer function (commonly termed activation or threshold function), and sent to the next layer in the network. An illustration of a single node is given in Fig. 3.2. The output $y$ of a given neuron is therefore found by

$$
y_{k}=\psi\left(\sum_{j=0}^{m} w_{k j} x_{j}\right)
$$

where $\psi$ is the activation function used by the neuron, $x$ is the input value from a nearby node, and $w$ is the weight of the connection between the nodes. Indices indicate that this output value is based on a summation of the values sent to the node. In the case of Fig. 3.2, the activation function shown is a simple step function that will cause the neuron to output either a positive or negative unit value depending on the sign of the summed inputs. The combination of a neural node and a binary step function is commonly referred to as a perceptron, and a layer of such couplings is known as a perceptron layer. A single perceptron exemplifies the most basic feed- 
forward ANN, and is capable of classifying linearly separable training data into two states. More complex ANNs - involving multiple nodes - are potentially capable of performing more complex pattern identification or classification tasks, as was illustrated by the examples cited in Section 3.1.

\subsection{Training}

The functionality of an ANN is accomplished by adjusting the weights of the connections between nodes based on supplied data - a task known as "training". The combinations of input and output (target) values used to train an ANN are referred to as training data, and they are vital in determining the final functionality and performance of the trained ANN. For each input-output combination in the training data, weights are updated according to one of several processes. An example backpropagation method functions as follows: input values are supplied to the input layer, and computations are propagated forward through the network with each neuron performing the computation shown in Fig. 3.2 (subject to the utilized activation functions) until values are output by the output layer of neurons. These computed output values are then compared to the target output values supplied in the training data - target values that correspond to the given input values that yielded the ANN output. If the values do not match, generally within some specified tolerance, weights are updated according to one of several algorithms. An example backpropagation algorithm is described by

$$
\begin{aligned}
& w_{i} \leftarrow w_{i}+\Delta w_{i} \\
& \Delta w_{i}=\eta(t-o) x_{i}
\end{aligned}
$$




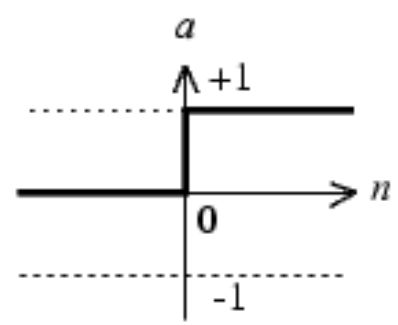

a.

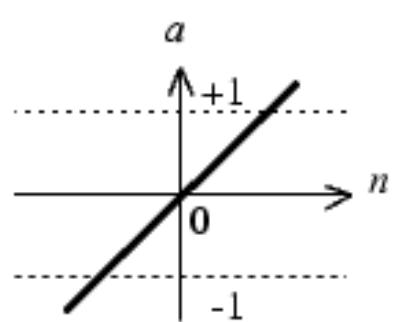

b

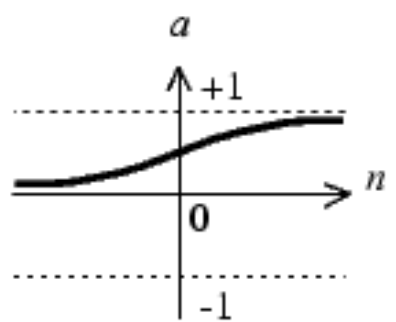

c

Figure 3.3: Common activation functions

where $w_{i}$ is weight of the connection to the $\mathrm{i}$-th neuron in the adjacent layer, $\Delta w_{i}$ is the change in weight calculated from the output and target mismatch, $\eta$ is a training rate parameter (often on the order of 0.1 ), $t$ is the target output value, $o$ is the computed output value, and $x_{i}$ is the value outputted by the i-th neuron along the connection weight ( $y_{k}$ in Eq. 3.1) [30]. It has been proven for numerous cases that by repeating this process for a number of training data combinations, the ANN will converge to the weights that allow the desired functionality; that is, when given a set of input values not included the training data, the ANN will return corresponding output values that agree with the pattern included in the training data [16]. This allows complex pattern recognition.

\subsection{Design}

Beyond the quantity and quality of supplied training data, there are several other parameters one can adjust in developing an ANN for a given application. The first is the pattern of connection between the layers of neurons. In the case of the common feed-forward ANN, as shown in Fig. 3.1, data is sequentially transmitted from the input layer through each hidden layer and finally to the output layer. Another possi- 
ble network organization involves interconnecting neurons between layers regardless of sequential order, creating a directed cycle. The second ANN parameter that can be adjusted is the activation function, several of which are shown in Fig. 3.3 [6]. The most simple activation function is a simple binary Heaviside step function (Fig. 3.3a), similar to the one utilized in Fig. 3.2. A second type of activation function, a linear activation function (shown in Fig. 3.3b), is capable of any output value, but is still limited to linearly separable training data. Despite this limitation, activation functions of this type are commonly used in linear filters. Another common activation function, the sigmoid transfer function (shown in Fig. 3.3c), can output any value between positive and negative infinity but normalizes the value between 0 and 1 .

A third adjustable ANN parameter is the learning algorithm used, which influences how weights are modified. ANNs are trained through a method in which the data supplied to the ANN includes inputs and their known target values, as described in Section 3.3. The task of the ANN is to identify a pattern relating input to target. A commonly-used example of a supervised learning method is the previously-discussed backpropagation algorithm. As a focus of this paper, it is worth describing two limitations of supervised learning. First, before desired functionality can be achieved, an adequate amount of accurate training data must first be provided. It is necessary to obtain a significant amount of data, often through experimentation, that accurately portrays the behavior of the system or pattern in question. The performance of the final trained ANN is therefore contingent on the quantity and quality of supplied training data, and requirements for both vary significantly depending on the specific task. Second, a trained ANN is generally incapable of applying a learned pattern to data that falls beyond the outer bounds of the supplied training data. Consequently, for an ANN to demonstrate accurate pattern recognition over a specified domain, the training data used to train the ANN must fully span the specified domain. 


\section{TRAINING AN ANN FOR SMA CHARACTERIZATION}

SMA phenomenological model parameter characterization is a task in which desired target values (the characteristic material parameters) are identified using input

data (typically stress-specific strain-temperature hysteresis data). With inputs and outputs that can be quantified prior to training, supervised learning of an ANN was selected as a feasible approach to facilitating the characterization process. As discussed in Chapter 3, however, this method requires extensive training data to obtain desirable modeling accuracy. To train an ANN for SMA material parameter characterization, it was decided that training data would be generated using an existing constitutive SMA model. Rather than using current characterization methods to generate experimentally-derived training data, a constitutive model-based approach to training data generation was selected here to allow more rapid assessment of this ANN-based approach.

\subsection{Model Implementation}

The goal of this thesis is to develop a method that yields an ANN capable of accurately recognizing the relationship between given strain-temperature data and corresponding SMA parameters. The Hartl-Lagoudas model introduced in Chapter 1 was chosen for implementation due to its demonstrated accuracy in modeling several aspects of the thermomechanical behavior of SMAs [11, 24]. Although this thesis focuses on pure tensile loading, the developed methodology could be applied to a case of pure shear loading with relative ease. Electing to focus on the case of uniaxial tensile force is therefore relatively inconsequential, as response to both tensile and shear loading is governed by the same fundamental principles. 
The full Hartl-Lagoudas model describes SMA behavior in three dimensions - for the full development and further information about this model, see [11]. In this thesis, a uniaxial reduction of the model is selected for use to ease implementation while remaining relevant to current SMA applications. In this reduction to one dimension, the 3 -D stress tensor simplifies to a single non-zero value, so $\bar{\sigma}$ becomes simply the original $\sigma_{11}$. Although the resulting strain tensor components are non-zero, the only component of interest is $\epsilon_{11}$. This simplifies the model to the variables $\sigma_{11}, \epsilon_{11}, x_{i}$, $T$, which can be solved using the 3-D equations described below.

The Hartl-Lagoudas SMA model is a three-dimensional and phenomenological model that focuses on the generation and recovery of strain within an SMA as a result of both forward and reverse solid-to-solid phase transformations. Three external state variables are considered: stress $(\sigma)$, transformation strain $(\epsilon)$, and temperature $(T)$. In addition to these, the model takes into account two internal state variables: inelastic transformation strain $\left(\epsilon^{t}\right)$ and martensitic volume fraction $(\xi)$. Temperature and total strain are assumed to be given, leaving three unknowns for calculation: $\sigma$, $\epsilon^{t}$, and $\xi$.

The constitutive model operates by evolving transformation strain given a loading history (in $\epsilon$ and $T$ ) and solving for stress-strain coupling while imposing constraints on the evolution of $\xi$. More specifically, computation involves first decomposing $\epsilon$ into elastic, thermal, and transformation components according to

$$
\epsilon=S(\xi) \sigma+\alpha\left(T-T_{0}\right)+\epsilon^{t}
$$

where $T_{0}$ is a reference initial temperature and $\alpha$ is the thermal expansion coefficient (a tensor) [13]. $S(\xi)$ is a compliance tensor that accounts for phase change according to 


$$
S(\xi)=S^{A}+\xi\left(S^{M}-S^{A}\right)
$$

where $S^{A}$ and $S^{M}$ are the austenitic and martensitic compliance tensors, respectively $[13]$.

The evolution of the inelastic transformation strain is found with

$$
\dot{\epsilon^{t}}=\dot{\xi}\left\{\begin{array}{cc}
H^{\text {cur }}(\bar{\sigma}) \frac{3}{2} \frac{\sigma^{\prime}}{\bar{\sigma}} & \dot{\xi}>0 \\
\frac{\epsilon^{t-r}}{\xi^{r}} & \dot{\xi}<0
\end{array}\right.
$$

Note that $\dot{\xi}>0$ indicates forward transformation, and $\dot{\xi}<0$ indicates reverse transformation. The von Mises equivalent stress given as $\bar{\sigma}=\sqrt{3 / 2 \sigma^{\prime}: \sigma^{\prime}}$, and $\sigma^{\prime}$ is the deviatoric stress. The magnitude of $\epsilon^{t}$ generated during forward transformation is driven by $H^{c u r}(\bar{\sigma})$, a scalar function that calculates the full transformation strain at the current stress level [15]. It is assumed that all transformation strain after forward transformation, at which point $\epsilon^{t}=\epsilon^{t-r}$ and $\xi=\xi^{r}$, is recovered if the reverse transformation returns the material to pure austenite $(\xi=0)$. To impose constraints on the evolution of $\epsilon^{t}$, the transformation function $\Phi^{t}$ is introduced along with the following constraints:

$$
\Phi^{t} \leq 0, \quad \dot{\xi} \Phi^{t}=0, \quad 0 \leq \xi \leq 1
$$

The transformation function is defined as two values, $\Phi_{f w d}^{t}$ and $\Phi_{r e v}^{t}$, for forward transformation and reverse transformation, respectively. These are defined as follows

$$
\begin{aligned}
\Phi_{f w d}^{t}= & (1-D) H^{c u r}(\bar{\sigma}) \bar{\sigma}+\frac{1}{2} \sigma: \tilde{S} \sigma+\rho \tilde{s}_{0} T-\rho \tilde{u}_{0} \\
& -\left[\frac{1}{2} a_{1}\left(1+\xi^{n_{1}}+(1-\xi)^{n_{2}}\right)+a_{3}\right]-Y_{0}^{t}
\end{aligned}
$$




$$
\begin{aligned}
\Phi_{r e v}^{t}= & -(1-D) \frac{\sigma: \epsilon^{t-r}}{\xi^{r}}-\frac{1}{2} \sigma: \tilde{S} \sigma-\rho \tilde{s}_{0} T+\rho \tilde{u}_{0} \\
& +\left[\frac{1}{2} a_{2}\left(1+\xi^{n_{3}}+(1-\xi)^{n_{4}}\right)-a_{3}\right]-Y_{0}^{t}
\end{aligned}
$$

where $D$ and $Y_{0}^{t}$ are transformation dissipation parameters, and $\rho \tilde{s_{0}}$ and $\rho \tilde{u_{0}}$ are parameters accounting for change in entropy and internal energy, respectively, between the two phases [11]. Transformation hardening coefficients are defined as $a_{1}, a_{2}$, and $a_{3}$, and transformation hardening exponents are defined as $n_{1}, n_{2}, n_{3}$, and $n_{4}$.

Implementation of the uniaxial model for this thesis was achieved through the creation of a script calling forward and reverse transformation functions. This, as well as all other instances of coding in this thesis, was done using MATLAB. The SMA model algorithm was verified by re-creating a given example in the source literature [11] - the resulting comparison is shown in Fig. 4.1.

\subsection{Training Data Generation}

Implementation of a constitutive SMA model allows generation of strain-temperature hysteresis plots using SMA parameters as inputs. The desired use of the trained ANN is to essentially reverse this process, outputting the SMA parameters using straintemperature hysteresis data as inputs. By using this constitutive model for training data generation, the target values necessary for training such a network are obtainable by simply storing the desired parameters used to create each hysteresis plot. Converting the generated hysteresis plots to ANN input values, however, requires an additional step of processing - each hysteresis plot is simplified into six pairs of strain-temperature coordinates. These coordinates are shown for an example strain- 

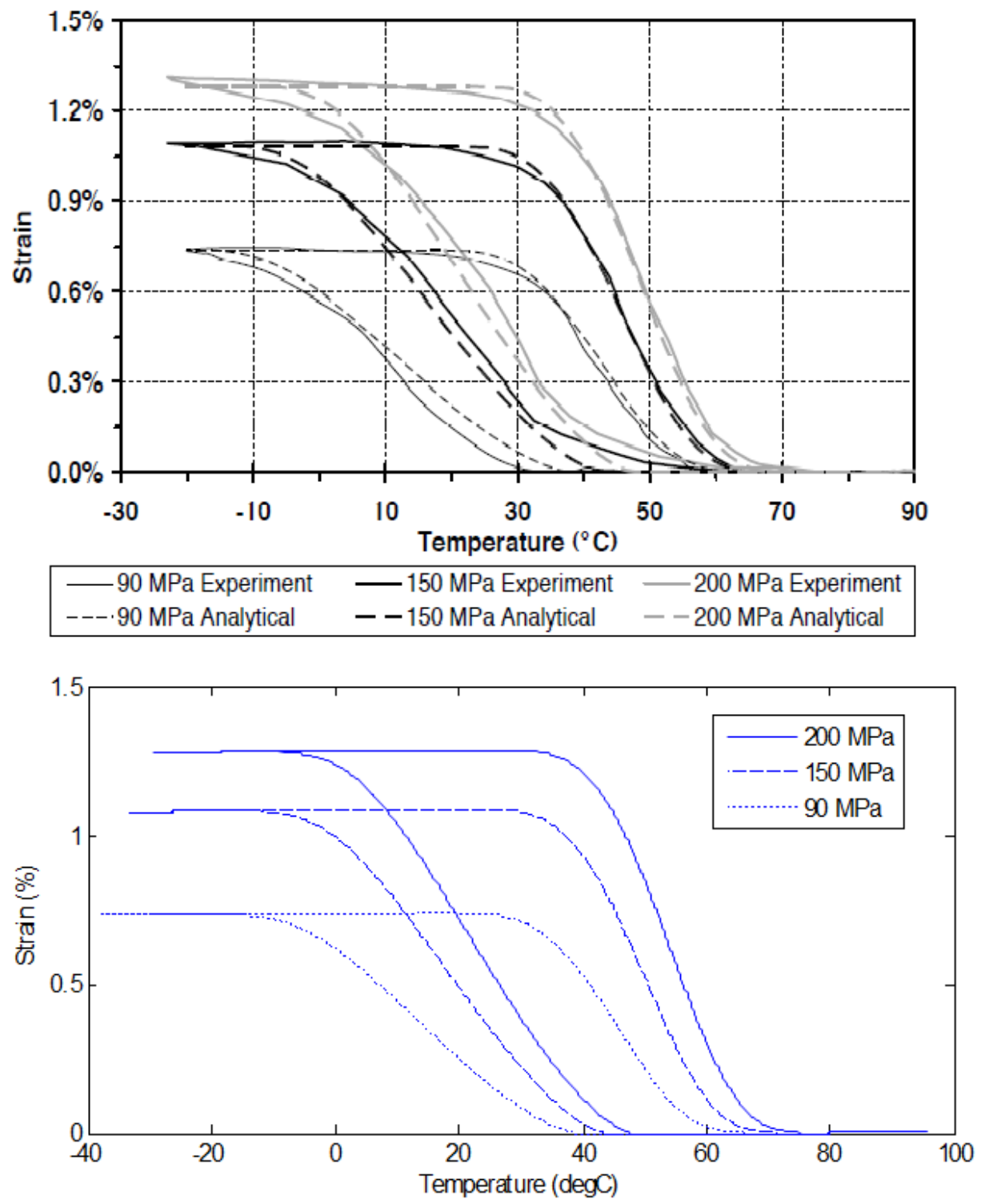

Figure 4.1: Model validations - literature example (top) versus implementation example (bottom) 


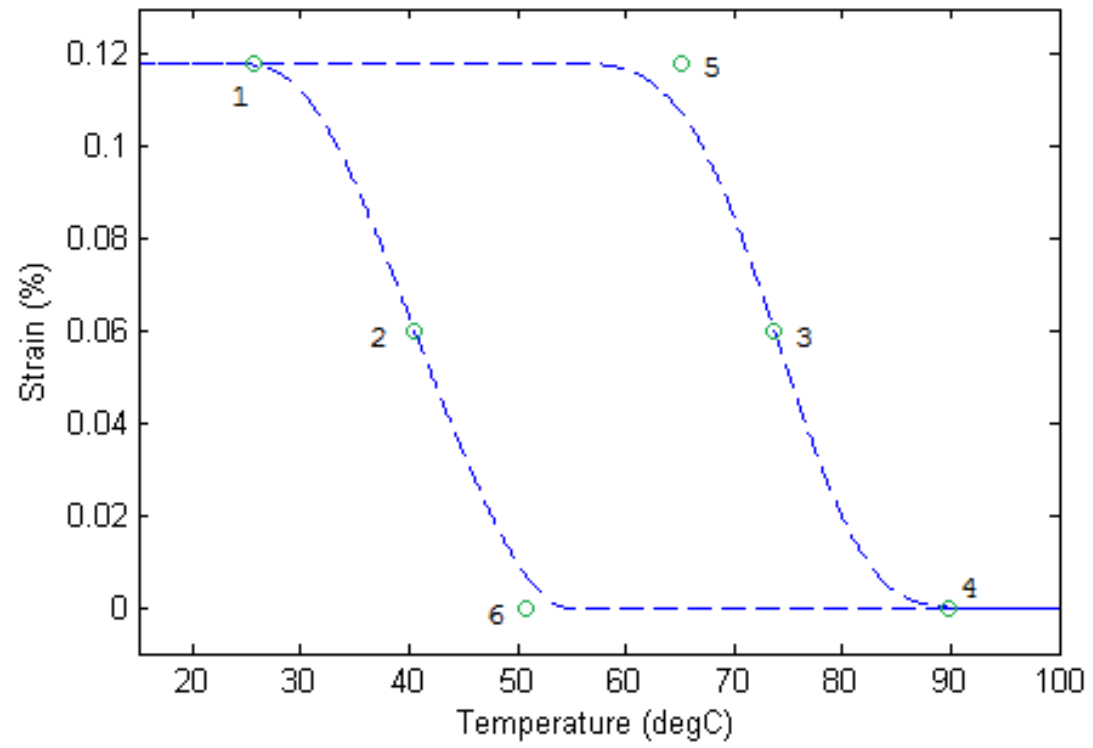

Figure 4.2: Hysteresis coordinate selection for ANN input

temperature plot in Fig. 4.2. A function is written to identify these points to allow for rapid training data generation. Points 1 and 4 mark the points at which the forward and reverse transformation paths converge within a specified strain tolerance. Points 2 and 3 are placed at the forward and reverse transformation coordinates corresponding to strain values halfway between the minimum and maximum strains. Point 5 is located at the intersection of a line passing through and with the same local slope as at Point 1 and a line similarly passing through and with the same local slope as at Point 3. Point 6 is found similarly but instead with Points 2 and 4 .

\subsubsection{Factorial Method}

With the goal of training an ANN to identify material parameters for a given SMA specimen, the training data must cover a wide range of SMA material parameter variation. At the same time, it is desired that the total number of theoretical SMAs used for training be kept relatively low-ideally less than 1000. This is selected 
as a rough constraint in this thesis largely to facilitate rapid evaluation of different approaches to training data generation, but also to assess the feasibility of repeating the process with experimental data. The amount of training data generated for each trained ANN was driven entirely by the selection of SMA parameter variation for the training specimens. For instance, if an SMA was characterized using just $A_{s}$, an ANN could be trained to identify the value of $A_{s}$ using data including several different values of the single parameter within an expected range. The quality of the trained ANN would depend on the number of different values of $A_{s}$ included in the training data. A "parameter resolution" of ten, as termed in this thesis, would yield ten specimens in the ANN training data. If an SMA was characterized with an additional parameter, such as $A_{f}$, training data would have to include different values of $A_{f}$ for an ANN to be able to identify both parameters. If one wanted to include a parameter resolution of ten for both, the total number of samples in the training data would equal $10^{2}$, or 100 . Using the uniaxial simplification of the Hartl-Lagoudas model, the behavior of an SMA is governed by over a dozen material parameters. Using a parameter resolution of ten with this number of parameters, the resulting training data would include $10^{15}$ samples - a largely impractical number when considering the case of an experimental data-based ANN. Even with a resolution of two for each parameter, the number of training specimens would approach 33,000 - a value much greater than the targeted maximum of 1000. This "factorial method" of training specimen SMA parameter selection, in which every possible combination of specified training specimen SMA parameter values is created, serves as a starting point for analysis in this work.

In the process of setting up a factorial-based method of training parameter values, a number of constraints are implemented that instruct the data generation functions to discard any samples that include physically impossible parameter values. With an 
understanding of transformation temperatures, it is evident that $A_{f}$ must be greater than $A_{s}$; likewise, $M_{f}$ must be less than $M_{s}$, and so on. These rules eliminate some percentage of generated training data samples because of the overlapping bounds of the four transformation temperatures. Implementation of these constraints thereby serves the double purpose of ensuring that the included training specimens are physically realizable and consequently reducing the number of training specimens. These constraints are therefore implemented in later methods of training specimen parameter generation.

Another step taken in implementing the factorial-based method is the prioritization of material parameters based on their influence of SMA thermomechanical behavior - i.e., training data should emphasize and contain more examples of a parameter with a wide range of possible values that significantly shapes the behavior of the system than a parameter with small variation that does not as significantly affect behavior. As discussed in Section 2.2, SMA transformation temperatures $\left(A_{s}\right.$, $\left.A_{f}, M_{s}, M_{f}\right)$ and stress influence coefficients $\left(C^{M}, C^{A}\right)$ are the material parameters targeted for identification in this work. As such, it is a given that variation of these parameters in the training data will take a high priority. However, this does not necessarily mean that all other parameters should be completely ignored, i.e., left with constant values in all training specimens. Including some variation of non-targeted parameters - especially parameters with a wide range of typical values - adds realism to the generated training data. For this reason, a few parameters are given a variety of values in training specimens: austenite and martensite Young's moduli $\left(E_{A}\right.$ and $\left.E_{M}\right)$, starting temperature $\left(T_{0}\right)$, and max transformation strain $\left(H_{\max }\right)$. A number of remaining SMA parameters are held at constant values. Based on the close-to-zero thermal expansion coefficient $(\alpha)$ values seen in the experimental data used to validate the SMA model in [11], $\alpha$ was fixed at a value of zero. Both $H_{m i n}$ 
Table 4.1: Training Data Parameter Values Bounds

\begin{tabular}{ccc}
\hline Parameter & Values & Units \\
\hline$T_{0}$ & 150,200 & ${ }^{\circ} \mathrm{C}$ \\
$E_{A}$ & 30,70 & $\mathrm{GPa}$ \\
$E_{M}$ & 30,70 & $\mathrm{GPa}$ \\
$\alpha$ & 0 & $1 /{ }^{\circ} \mathrm{C}$ \\
$M_{s}$ & 24,90 & ${ }^{\circ} \mathrm{C}$ \\
$M_{f}$ & $-20,40$ & ${ }^{\circ} \mathrm{C}$ \\
$A_{s}$ & 10,70 & ${ }^{\circ} \mathrm{C}$ \\
$A_{f}$ & 50,120 & ${ }^{\circ} \mathrm{C}$ \\
$C^{M}$ & 8,18 & $\mathrm{MPa} /{ }^{\circ} \mathrm{C}$ \\
$C^{A}$ & 8,18 & $\mathrm{MPa} /{ }^{\circ} \mathrm{C}$ \\
$H_{\max }$ & $0.04,0.07$ & - \\
$H_{\min }$ & 0 & - \\
$k$ & 200 & - \\
$\sigma_{\text {crit }}$ & 0 & $\mathrm{~Pa}$ \\
\hline
\end{tabular}

and $k_{\text {exp }}$ were similarly fixed at reasonable values based on examples given in [11]. As prefaced, the decision to leave a few SMA parameters as fixed values through all training specimens is made after taking into consideration the relative influences of the various parameters on SMA behavior. This decision is further justified by the focus of this work: the development of a methodology for training an ANN for SMA characterization. If the developed methodology produces an ANN capable of identifying material parameters for data somewhat simplified by judicious selection of training specimen parameter variation, it stands to reason that the method could be repeated with additional training data for a more complex system.

Outer bounds for material parameter variation are specified, and a baseline factorial-based training data set is generated using the parameter bounds - shown 
Table 4.2: Training Data Parameter Values Using Factorial Method

\begin{tabular}{ccc}
\hline Parameter & Values & Units \\
\hline$T_{0}$ & 150,200 & ${ }^{\circ} \mathrm{C}$ \\
$E_{A}$ & 30,70 & $\mathrm{GPa}$ \\
$E_{M}$ & 30,70 & $\mathrm{GPa}$ \\
$\alpha$ & 0 & $1 /{ }^{\circ} \mathrm{C}$ \\
$M_{s}$ & $24,60,90$ & ${ }^{\circ} \mathrm{C}$ \\
$M_{f}$ & $-20,10,40$ & ${ }^{\circ} \mathrm{C}$ \\
$A_{s}$ & $10,40,70$ & ${ }^{\circ} \mathrm{C}$ \\
$A_{f}$ & $50,85,120$ & ${ }^{\circ} \mathrm{C}$ \\
$C^{M}$ & $8,13,18$ & $\mathrm{MPa} /{ }^{\circ} \mathrm{C}$ \\
$C^{A}$ & $8,13,18$ & $\mathrm{MPa} /{ }^{\circ} \mathrm{C}$ \\
$H_{\text {max }}$ & $0.04,0.07$ & - \\
$H_{\text {min }}$ & 0 & - \\
$k$ & 200 & - \\
$\sigma_{\text {crit }}$ & 0 & $\mathrm{~Pa}$ \\
\hline
\end{tabular}

in Table 4.1. The bounds in this case are selected based on typical values found throughout the literature and are intended to include a wide enough range to allow training of an ANN that can identify material properties for a variety of SMA specimens. The parameter selection shown in Figure 4.1 yield 360 training specimens. To allow an analysis of ANN accuracy versus number of training specimens, additional included values are sequentially added at approximate midpoints between the outer bounds specified in Table 4.1 for $M_{s}, M_{f}, A_{s}, A_{f}, C^{M}$, and $C^{A}$. This leads to the final listing of included training specimen parameter values shown in Table 4.2, which yields 4296 training specimens.

To capture the effect of stress influence coefficients, hysteresis data is generated at two stress values (50 $\mathrm{MPa}$ and $400 \mathrm{MPa}$ ) for each set of SMA parameters. An 


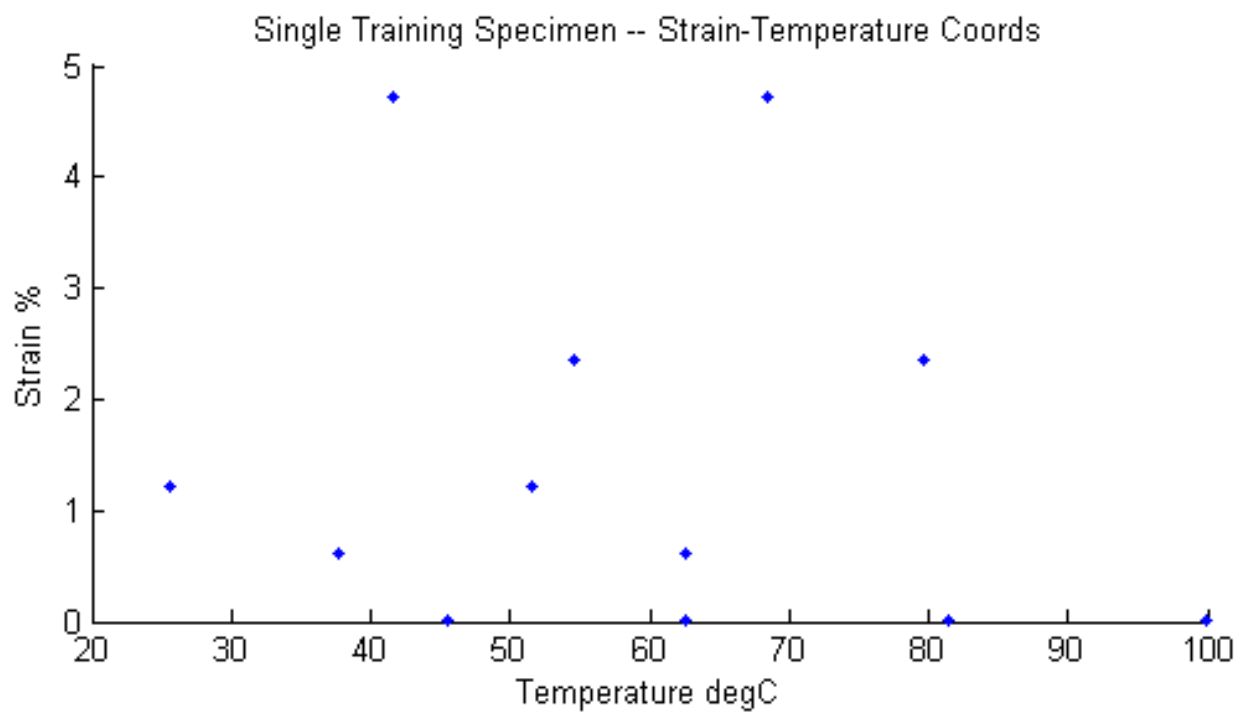

Figure 4.3: Strain-temperature coordinates for a single training specimen

example of ANN input strain-temperature coordinates for a single training specimen are shown in Figure 4.3. As shown, each specimen is seen through the eyes of the ANN as twelve strain-temperature coordinates - six coordinates describing the hysteresis plots generated at the two specified constant stress values. A visualization of a full training input data set-showing all ANN input hysteresis coordinates - is shown in Figure 4.4. In this case (Figure 4.4), this is the input training data corresponding to the parameters bounds given in Table 4.1, which yielded 360 training specimens. Using the specified outer bounds of the training data parameter values, Figure 4.4 therefore shows the bounds of the full strain-temperature domain explored in this thesis. For comparison, a visualization of the 4296 training specimens generated by Table 4.2 is presented in Figure 4.5.

\subsubsection{Taguchi Method}

After following the factorial approach described in Section 4.2.1, in which every possible combination of selected SMA parameter values is included, the task of 


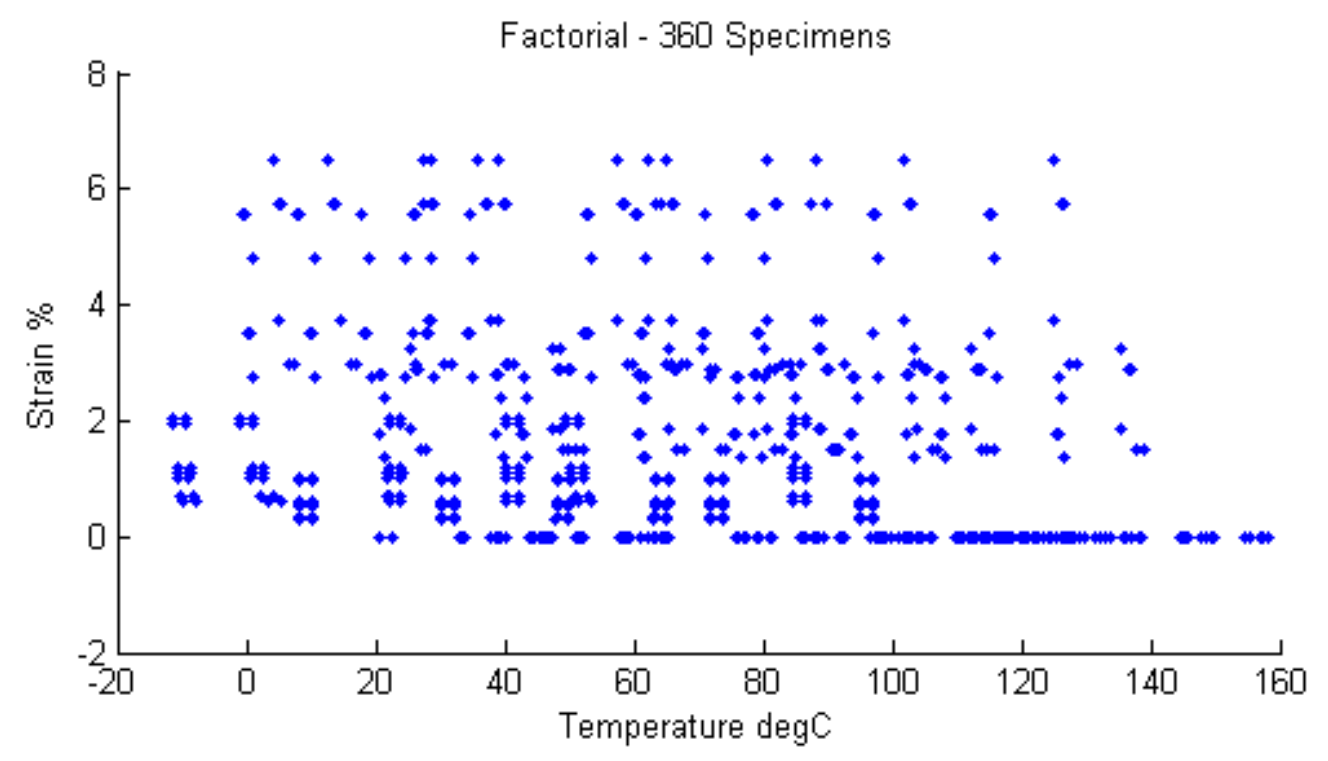

Figure 4.4: Full training data set containing 360 specimens

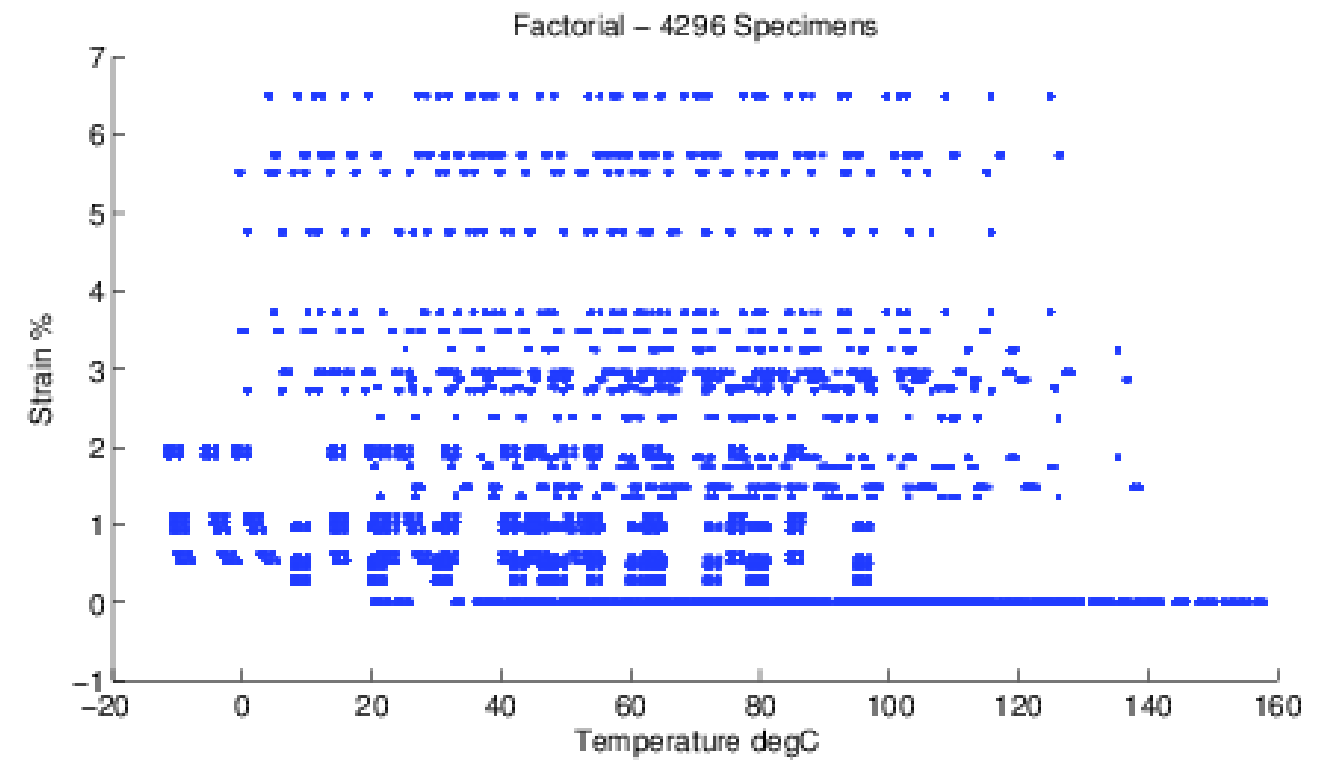

Figure 4.5: Full training data set containing 4296 specimens 
training data generation was re-approached using Taguchi methods. Taguchi methods [32] are a group of statistical methods originally developed with the intent of improving the efficiency of manufacturing processes. They have since been applied in a number of different contexts and have been found to be particularly useful in experiment design $[36,8,38]$.

When approaching experiment design using a generic factorial method, the goal is to examine every possible combination of every given test parameter. For example, three test parameters, each having two possible values, would necessitate $2^{3}$ experiments - a total of eight experiments. When applying Taguchi methods to experiment design, however, the goal is to merely examine every possible combination of any given pair of test parameters. Applying this approach to the above example yields a total of four experiments, shown in Table 4.3. In this simple case, the application of Taguchi methods reduces the number of experiments required to explore the system dynamics from eight to four - a $50 \%$ reduction. This reduction percentage is dramatically increased as the number of system parameters and possible parameter values are increased. Moving from three to seven parameters with two possible values each, a factorial approach would call for $2^{7}$ or 128 experiments. Using a Taguchi approach outlined in Table 4.4, this is reduced to a total of eight experiments - a 93.75\% reduction. As a final example, in the case of considering 13 system parameters with three possible values each, a factorial approach to exploring the full range of system dynamics would necessitate for $3^{13}$ or 1,594,323 experiments. A Taguchi approach here would call for only 27 experiments as shown in Table 4.5-a 99.999\% reduction.

Ostensibly, this approach allows one to evaluate how each parameter influences a given system using fewer experiments than is necessary when following the factorial approach. The ability to contain a wide range of system variations within a small 
Table 4.3: Taguchi Method Application: Example 1

\begin{tabular}{cccc}
\hline Experiment & P1 & P2 & P3 \\
\hline 1 & 1 & 1 & 1 \\
2 & 1 & 2 & 2 \\
3 & 2 & 1 & 2 \\
4 & 2 & 2 & 1 \\
\hline
\end{tabular}

Table 4.4: Taguchi Method Application: Example 2

\begin{tabular}{cccccccc}
\hline Experiment & P1 & P2 & P3 & P4 & P5 & P6 & P7 \\
\hline 1 & 1 & 1 & 1 & 1 & 1 & 1 & 1 \\
2 & 1 & 1 & 1 & 2 & 2 & 2 & 2 \\
3 & 1 & 2 & 2 & 1 & 1 & 2 & 2 \\
4 & 1 & 2 & 2 & 2 & 2 & 1 & 1 \\
5 & 2 & 1 & 2 & 1 & 2 & 1 & 2 \\
6 & 2 & 1 & 2 & 2 & 1 & 2 & 1 \\
7 & 2 & 2 & 1 & 1 & 2 & 2 & 1 \\
8 & 2 & 2 & 1 & 2 & 1 & 1 & 2 \\
\hline
\end{tabular}


Table 4.5: Taguchi Method Application: Example 3

\begin{tabular}{cccccccccccccc}
\hline Experiment & P1 & P2 & P3 & P4 & P5 & P6 & P7 & P8 & P9 & P10 & P11 & P12 & P13 \\
\hline 1 & 1 & 1 & 1 & 1 & 1 & 1 & 1 & 1 & 1 & 1 & 1 & 1 & 1 \\
2 & 1 & 1 & 1 & 1 & 2 & 2 & 2 & 2 & 2 & 2 & 2 & 2 & 2 \\
3 & 1 & 1 & 1 & 1 & 3 & 3 & 3 & 3 & 3 & 3 & 3 & 3 & 3 \\
4 & 1 & 2 & 2 & 2 & 1 & 1 & 1 & 2 & 2 & 2 & 3 & 3 & 3 \\
5 & 1 & 2 & 2 & 2 & 2 & 2 & 2 & 3 & 3 & 3 & 1 & 1 & 1 \\
6 & 1 & 2 & 2 & 2 & 3 & 3 & 3 & 1 & 1 & 1 & 2 & 2 & 2 \\
7 & 1 & 3 & 3 & 3 & 1 & 1 & 1 & 3 & 3 & 3 & 2 & 2 & 2 \\
8 & 1 & 3 & 3 & 3 & 2 & 2 & 2 & 1 & 1 & 1 & 3 & 3 & 3 \\
9 & 1 & 3 & 3 & 3 & 3 & 3 & 3 & 2 & 2 & 2 & 1 & 1 & 1 \\
10 & 2 & 1 & 2 & 3 & 1 & 2 & 3 & 1 & 2 & 3 & 1 & 2 & 3 \\
11 & 2 & 1 & 2 & 3 & 2 & 3 & 1 & 2 & 3 & 1 & 2 & 3 & 1 \\
12 & 2 & 1 & 2 & 3 & 3 & 1 & 2 & 3 & 1 & 2 & 3 & 1 & 2 \\
13 & 2 & 2 & 3 & 1 & 1 & 2 & 3 & 2 & 3 & 1 & 3 & 1 & 2 \\
14 & 2 & 2 & 3 & 1 & 2 & 3 & 1 & 3 & 1 & 2 & 1 & 2 & 3 \\
15 & 2 & 2 & 3 & 1 & 3 & 1 & 2 & 1 & 2 & 3 & 2 & 3 & 1 \\
16 & 2 & 3 & 1 & 2 & 1 & 2 & 3 & 3 & 1 & 2 & 2 & 3 & 1 \\
17 & 2 & 3 & 1 & 2 & 2 & 3 & 1 & 1 & 2 & 3 & 3 & 1 & 2 \\
18 & 2 & 3 & 1 & 2 & 3 & 1 & 2 & 2 & 3 & 1 & 1 & 2 & 3 \\
19 & 3 & 1 & 3 & 2 & 1 & 3 & 2 & 1 & 3 & 2 & 1 & 3 & 2 \\
20 & 3 & 1 & 3 & 2 & 2 & 1 & 3 & 2 & 1 & 3 & 2 & 1 & 3 \\
21 & 3 & 1 & 3 & 2 & 3 & 2 & 1 & 3 & 2 & 1 & 3 & 2 & 1 \\
22 & 3 & 2 & 1 & 3 & 1 & 3 & 2 & 2 & 1 & 3 & 3 & 2 & 1 \\
23 & 3 & 2 & 1 & 3 & 2 & 1 & 3 & 3 & 2 & 1 & 1 & 3 & 2 \\
24 & 3 & 2 & 1 & 3 & 3 & 2 & 1 & 1 & 3 & 2 & 2 & 1 & 3 \\
25 & 3 & 3 & 2 & 1 & 1 & 3 & 2 & 3 & 2 & 1 & 2 & 1 & 3 \\
26 & 3 & 3 & 2 & 1 & 2 & 1 & 3 & 1 & 3 & 2 & 3 & 2 & 1 \\
27 & 3 & 3 & 2 & 1 & 3 & 2 & 1 & 2 & 1 & 3 & 1 & 3 & 2 \\
\hline
\end{tabular}


data set was desirable in the context of this work because it potentially reduces the amount of training data required to train an adequate ANN for the purpose of SMA material parameter identification. The exact means of applying Taguchi methods to SMA training data, however, was not as simple as the above examples. As previously described, the accuracy of a trained ANN significantly depends on the quality and quantity of the data used to train it. For this reason, it is still necessary for the training data generated using Taguchi methods to contain a variety of different material parameters. As in the case of the factorial method, ten material parameters are chosen $\left(T_{0}, E_{A}, E_{M}, M_{s}, M_{f}, A_{s}, A_{f}, C^{M}, C^{A}, H^{\max }\right)$, and five possible values are specified for each parameter (see Figure 4.6. Using Taguchi methods, this setup outputs a maximum of fifty training specimens - the specific orthogonal array used in this case, an L50 orthogonal array, is set up for twelve parameters with up to five possible values each and is given in Table 4.8. The case of five possible parameter values serves as a baseline for implementation of the Taguchi-based approach, and the included parameter values are given in Table 4.6. To allow fair comparison between the Taguchi and factorial-based approaches to training data generation, the same material parameter outer bounds are used (recall Table 4.1) in the generation of training specimen material parameter values.

Similar to the gradual increase of training specimen parameters performed for the factorial-based case, additional parameter values are sequentially added to the Taguchi-based training specimens. Because the L50 Taguchi table expects parameters with five possible values, these additional values are added in groups of five, being used to increase the parameter resolution of the four transformation temperatures $\left(M_{s}, M_{f}, A_{s}, A_{f}\right)$. The final set of parameters used in the Taguchi-based approach to training data generation is given in Table 4.7.

The ten specified parameter values for the each transformation temperature are 
Table 4.6: Baseline Training Data Parameter Values using Taguchi-based Method

\begin{tabular}{ccc}
\hline Parameter & Values & Units \\
\hline$T_{0}$ & $150,160,170,180,200$ & ${ }^{\circ} \mathrm{C}$ \\
$E_{A}$ & $30,40,50,60,70$ & $\mathrm{GPa}$ \\
$E_{M}$ & $30,40,50,60,70$ & $\mathrm{GPa}$ \\
$\alpha$ & 0 & $1 /{ }^{\circ} \mathrm{C}$ \\
$M_{s}$ & $24,36,60,75,90$ & ${ }^{\circ} \mathrm{C}$ \\
$M_{f}$ & $-20,-5,10,25,40$ & ${ }^{\circ} \mathrm{C}$ \\
$A_{s}$ & $10,25,40,55,70$ & ${ }^{\circ} \mathrm{C}$ \\
$A_{f}$ & $50,70,85,105,120$ & ${ }^{\circ} \mathrm{C}$ \\
$C^{M}$ & $8,10,13,15,18$ & $\mathrm{MPa} /{ }^{\circ} \mathrm{C}$ \\
$C^{A}$ & $8,10,13,15,18$ & $\mathrm{MPa} /{ }^{\circ} \mathrm{C}$ \\
$H_{\max }$ & $0.04,0.045,0.050,0.060,0.07$ & - \\
$H_{\min }$ & 0 & - \\
$k$ & 200 & - \\
$\sigma_{\text {crit }}$ & 0 & $\mathrm{~Pa}$ \\
\hline
\end{tabular}

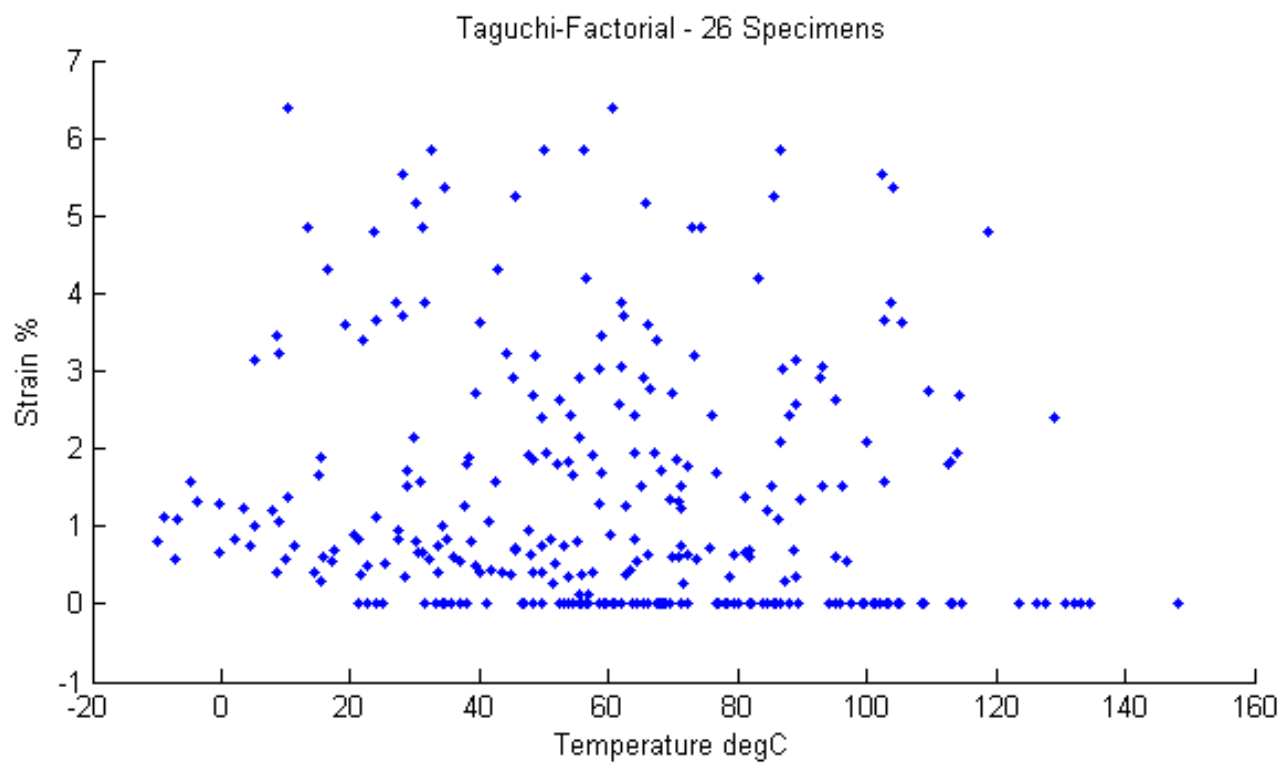

Figure 4.6: Baseline Taguchi-based training data set containing 26 specimens 
Table 4.7: Training Data Parameter Values using Taguchi-based Method

\begin{tabular}{ccc}
\hline Parameter & Values & Units \\
\hline$T_{0}$ & $150,160,170,180,200$ & ${ }^{\circ} \mathrm{C}$ \\
$E_{A}$ & $30,40,50,60,70$ & $\mathrm{GPa}$ \\
$E_{M}$ & $30,40,50,60,70$ & $\mathrm{GPa}$ \\
$\alpha$ & 0 & $1 /{ }^{\circ} \mathrm{C}$ \\
$M_{s}$ & $24,29,36,44,60,62,70,75,85,90$ & ${ }^{\circ} \mathrm{C}$ \\
$M_{f}$ & $-20,-14,-8,-5,2,10,19,25,31,40$ & ${ }^{\circ} \mathrm{C}$ \\
$A_{s}$ & $10,16,25,29,37,40,49,55,63,70$ & ${ }^{\circ} \mathrm{C}$ \\
$A_{f}$ & $50,57,64,70,78,85,97,105,112,120$ & ${ }^{\circ} \mathrm{C}$ \\
$C^{M}$ & $8,10,13,15,18$ & $\mathrm{MPa} /{ }^{\circ} \mathrm{C}$ \\
$C^{A}$ & $8,10,13,15,18$ & $\mathrm{MPa} /{ }^{\circ} \mathrm{C}$ \\
$H_{\max }$ & $0.04,0.045,0.050,0.060,0.07$ & - \\
$H_{\min }$ & 0 & - \\
$k$ & 200 & - \\
$\sigma_{\text {crit }}$ & 0 & $\mathrm{~Pa}$ \\
\hline
\end{tabular}


then divided into two sets of five. Training data is generated using the L50 Taguchi array for each combination of these sets of transformation temperature values. Using this method, the generated training data is able to contain ten values of each of the four transformation values and five of each of the remaining varied SMA parameters $\left(T_{0}, E_{A}, E_{M}, C^{M}, C^{A}, H_{\max }\right)$. These included values are shown in Table 4.7. Capturing this degree of parameter value variation using the factorial approach would necessitate up to 780 million training specimens $\left(10^{4} * 5^{7}\right)$. Using Taguchi methods, however, this is reduced to a more reasonable maximum of 800 training specimens $\left(2^{4} * 50\right)$. Because this data generation method involves applying Taguchi principles to multiple parameter sets in a factorial fashion, it is referred to as either the Taguchi or Taguchi-factorial method in this thesis. Assigning the material parameters $\left(M_{S}\right.$, $M_{f}$, etc.) to the L50 columns shown in Table 4.8 for the baseline case described in Table 4.6 yields 26 training specimens (physically impossible parameter combinations are discarded), which are visualized in Figure 4.6. Using the final parameter values specified in Table 4.7 yields 482 training specimens. A visualization of the ANN input strain-temperature coordinates for this data is given in Figure 4.7. 
Table 4.8: L50 Orthogonal Array

\begin{tabular}{|c|c|c|c|c|c|c|c|c|c|c|c|c|}
\hline Experiment & $\mathrm{P} 1$ & $\mathrm{P} 2$ & P3 & $\mathrm{P} 4$ & $\mathrm{P} 5$ & $\mathrm{P} 6$ & P7 & P8 & P9 & P10 & $\mathrm{P} 11$ & P12 \\
\hline 1 & 1 & 1 & 1 & 1 & 1 & 1 & 1 & 1 & 1 & 1 & 1 & 1 \\
\hline 2 & 1 & 1 & 2 & 2 & 2 & 2 & 2 & 2 & 2 & 2 & 2 & 2 \\
\hline 3 & 1 & 1 & 3 & 3 & 3 & 3 & 3 & 3 & 3 & 3 & 3 & 3 \\
\hline 4 & 1 & 1 & 4 & 4 & 4 & 4 & 4 & 4 & 4 & 4 & 4 & 4 \\
\hline 5 & 1 & 1 & 5 & 5 & 5 & 5 & 5 & 5 & 5 & 5 & 5 & 5 \\
\hline 6 & 1 & 2 & 1 & 2 & 3 & 4 & 5 & 1 & 2 & 3 & 4 & 5 \\
\hline 7 & 1 & 2 & 2 & 3 & 4 & 5 & 1 & 2 & 3 & 4 & 5 & 1 \\
\hline 8 & 1 & 2 & 3 & 4 & 5 & 1 & 2 & 3 & 4 & 5 & 1 & 2 \\
\hline 9 & 1 & 2 & 4 & 5 & 1 & 2 & 3 & 4 & 5 & 1 & 2 & 3 \\
\hline 10 & 1 & 2 & 5 & 1 & 2 & 3 & 4 & 5 & 1 & 2 & 3 & 4 \\
\hline 11 & 1 & 3 & 1 & 3 & 5 & 2 & 4 & 4 & 1 & 3 & 5 & 2 \\
\hline 12 & 1 & 3 & 2 & 4 & 1 & 3 & 5 & 5 & 2 & 4 & 1 & 3 \\
\hline 13 & 1 & 3 & 3 & 5 & 2 & 4 & 1 & 1 & 3 & 5 & 2 & 4 \\
\hline 14 & 1 & 3 & 4 & 1 & 3 & 5 & 2 & 2 & 4 & 1 & 3 & 5 \\
\hline 15 & 1 & 3 & 5 & 2 & 4 & 1 & 3 & 3 & 5 & 2 & 4 & 1 \\
\hline 16 & 1 & 4 & 1 & 4 & 2 & 5 & 3 & 5 & 3 & 1 & 4 & 2 \\
\hline 17 & 1 & 4 & 2 & 5 & 3 & 1 & 4 & 1 & 4 & 2 & 5 & 3 \\
\hline 18 & 1 & 4 & 3 & 1 & 4 & 2 & 5 & 2 & 5 & 3 & 1 & 4 \\
\hline 19 & 1 & 4 & 4 & 2 & 5 & 3 & 1 & 3 & 1 & 4 & 2 & 5 \\
\hline 20 & 1 & 4 & 5 & 3 & 1 & 4 & 2 & 4 & 2 & 5 & 3 & 1 \\
\hline 21 & 1 & 5 & 1 & 5 & 4 & 3 & 2 & 4 & 3 & 2 & 1 & 5 \\
\hline 22 & 1 & 5 & 2 & 1 & 5 & 4 & 3 & 5 & 4 & 3 & 2 & 1 \\
\hline 23 & 1 & 5 & 3 & 2 & 1 & 5 & 4 & 1 & 5 & 4 & 3 & 2 \\
\hline 24 & 1 & 5 & 4 & 3 & 2 & 1 & 5 & 2 & 1 & 5 & 4 & 3 \\
\hline 25 & 1 & 5 & 5 & 4 & 3 & 2 & 1 & 3 & 2 & 1 & 5 & 4 \\
\hline 26 & 2 & 1 & 1 & 1 & 4 & 5 & 4 & 3 & 2 & 5 & 2 & 3 \\
\hline 27 & 2 & 1 & 2 & 2 & 5 & 1 & 5 & 4 & 3 & 1 & 3 & 4 \\
\hline 28 & 2 & 1 & 3 & 3 & 1 & 2 & 1 & 5 & 4 & 2 & 4 & 5 \\
\hline 29 & 2 & 1 & 4 & 4 & 2 & 3 & 2 & 1 & 5 & 3 & 5 & 1 \\
\hline 30 & 2 & 1 & 5 & 5 & 3 & 4 & 3 & 2 & 1 & 4 & 1 & 2 \\
\hline 31 & 2 & 2 & 1 & 2 & 1 & 3 & 3 & 2 & 4 & 5 & 5 & 4 \\
\hline 32 & 2 & 2 & 2 & 3 & 2 & 4 & 4 & 3 & 5 & 1 & 1 & 5 \\
\hline 33 & 2 & 2 & 3 & 4 & 3 & 5 & 5 & 4 & 1 & 2 & 2 & 1 \\
\hline 34 & 2 & 2 & 4 & 5 & 4 & 1 & 1 & 5 & 2 & 3 & 3 & 2 \\
\hline 35 & 2 & 2 & 5 & 1 & 5 & 2 & 2 & 1 & 3 & 4 & 4 & 3 \\
\hline 36 & 2 & 3 & 1 & 3 & 3 & 1 & 2 & 5 & 5 & 4 & 2 & 4 \\
\hline 37 & 2 & 3 & 1 & 3 & 3 & 1 & 2 & 5 & 5 & 4 & 2 & 4 \\
\hline 38 & 2 & 3 & 3 & 5 & 5 & 3 & 4 & 2 & 2 & 1 & 4 & 1 \\
\hline 39 & 2 & 3 & 4 & 1 & 1 & 4 & 5 & 3 & 3 & 2 & 5 & 2 \\
\hline 40 & 2 & 3 & 5 & 2 & 2 & 5 & 1 & 4 & 4 & 3 & 1 & 3 \\
\hline 41 & 2 & 4 & 1 & 4 & 5 & 4 & 1 & 2 & 5 & 2 & 3 & 3 \\
\hline 42 & 2 & 4 & 2 & 5 & 1 & 5 & 2 & 3 & 1 & 3 & 4 & 4 \\
\hline 43 & 2 & 4 & 3 & 1 & 2 & 1 & 3 & 4 & 2 & 4 & 5 & 5 \\
\hline 44 & 2 & 4 & 4 & 2 & 3 & 2 & 4 & 5 & 3 & 5 & 1 & 1 \\
\hline 45 & 2 & 4 & 5 & 3 & 4 & 3 & 5 & 1 & 4 & 1 & 2 & 3 \\
\hline 46 & 2 & 5 & 1 & 5 & 2 & 2 & 5 & 3 & 4 & 4 & 3 & 1 \\
\hline 47 & 2 & 5 & 2 & 1 & 3 & 3 & 1 & 4 & 5 & 5 & 4 & 2 \\
\hline 48 & 2 & 5 & 3 & 2 & 4 & 4 & 2 & 5 & 1 & 1 & 5 & 3 \\
\hline 49 & 2 & 5 & 4 & 3 & 5 & 5 & 3 & 1 & 2 & 2 & 1 & 4 \\
\hline 50 & 2 & 5 & 5 & 4 & 1 & 1 & 4 & 2 & 3 & 3 & 2 & 5 \\
\hline
\end{tabular}




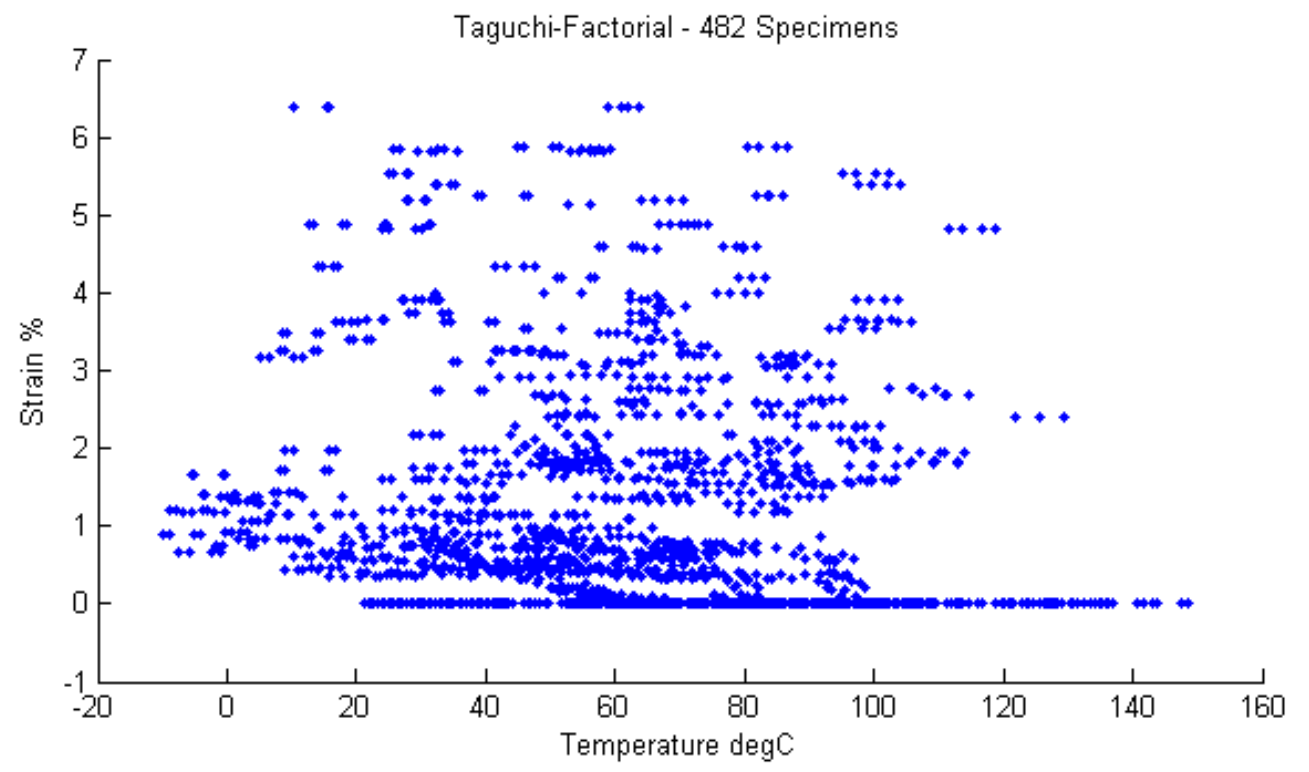

Figure 4.7: Full taguchi-based training data set containing 482 specimens 


\section{RESULTS}

Numerous sets of training data were generated in the process of finding a balance between ANN characterization accuracy and the number of training data specimens. In this iterative process, an ANN was trained for each set of training data, and its characterization accuracy was then assessed. New training data were then generated based on the performance of the ANN. Results for select iterations of this process - typically first and final iterations - are given in this chapter. This process was repeated using both a factorial and Taguchi-based approach to training data generation, as discussed in Section 4.2. A preliminary study focused on comparing the performance of ANNs trained with strain-temperature data corresponding to either one or two cases of constant applied stress. From here, the focus became the comparison of the ANN performances corresponding to the factorial and Taguchi-factorial methods of training data generation.

To facilitate the performance evaluation of the trained ANNs, a MATLAB script was created that generates a specified number of random theoretical SMA specimens with material parameters falling within the bounds of the training data. ANN performance evaluation was performed using 100 such specimens unless otherwise specified. Strain-temperature coordinates for these specimens are then generated at 50 and $400 \mathrm{MPa}$ and then given, along with $T_{0}$, to the trained $\mathrm{ANN}$ as inputs. The ANN output values - predicted material parameter values - are then compared with the known values to calculate error. This process is included in Figure 1.6. 


\subsection{ANN Structure and Training}

ANN creation and training was performed through use of the MATLAB Neural Network toolbox. Though some experimentation was conducted regarding the ANN parameters described in Chapter 3, default toolbox options were largely selected. A simple preliminary study indicated that characterization accuracy was largely unaffected by the number of hidden layer nodes included in the ANN structure as

long as more than four hidden layer nodes were used. The results of this study are summarized in Figure 5.1.

The final standardized ANN design used in generating results presented in this chapter included twenty-five input nodes, a hidden layer consisting of twenty neurons, and six output neurons. The hidden layer neurons used a sigmoid transfer function, and those of the output layer used a linear transfer function. Of the given training data samples, seventy percent were randomly selected for training, being applied to the network and causing changes in weights. Of the remaining data, half was used for validation - used to evaluate the performance of the ANN and halt training if a target performance criterion is met. The final fifteen percent of the training data was used for testing - used to independently evaluate the performance of the ANN after training has concluded. The Levenberg-Marquardt algorithm was selected as the training method - a popular numerical optimization technique often used in curvefitting problems. Performance of the ANN during training was evaluated using mean squared error.

\subsection{One-Plot Method vs. Two-Plot Method}

A second preliminary study assessed how ANNs trained using strain-temperature coordinates for two cases of applied stress performed compared to cases involving 


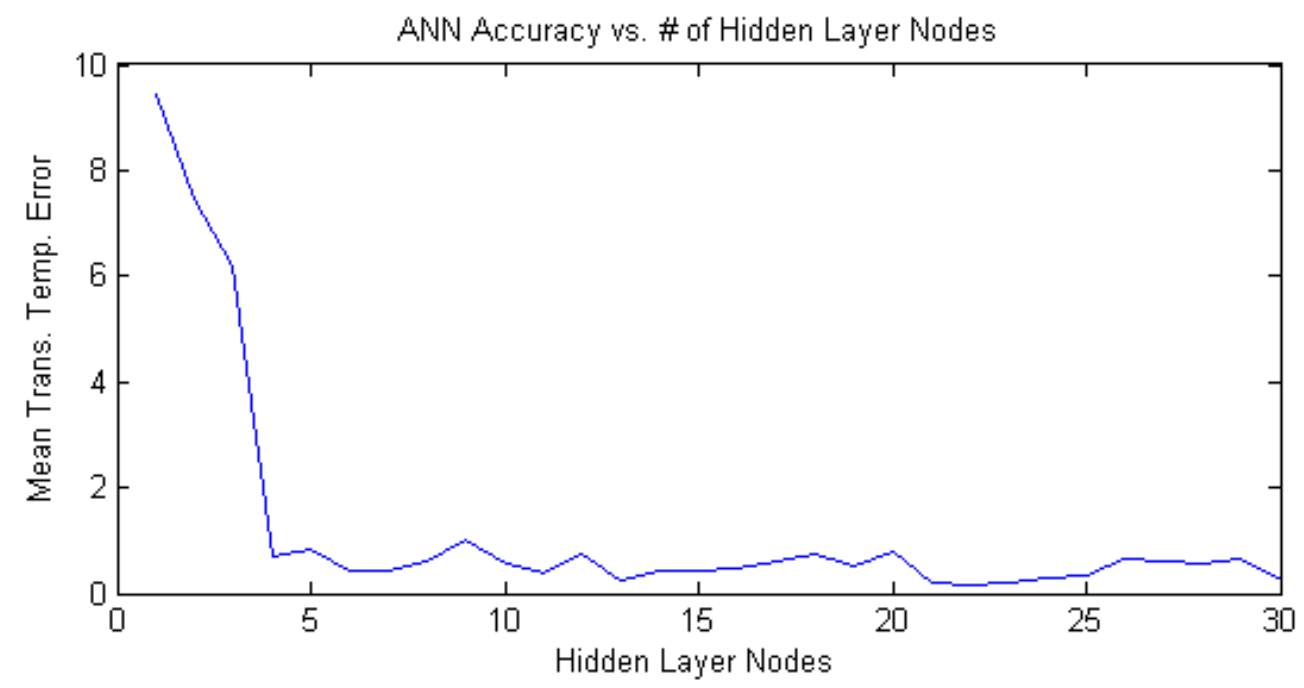

Figure 5.1: Study assessing ANN accuracy vs number of included hidden layer nodes

a single value of applied stress - termed "two-plot" and "one-plot" methods. To illustrate the method of performance evaluation, a single evaluation example is given in Tables 5.1 and 5.2. Table 5.1 shows the parameters of a SMA specimen used to evaluate the characterization accuracy of a trained ANN, and Table 5.2 compares the identification of these parameters using two different ANNs. Subtracting ANN output from the target value for each parameter allows calculation of ANN prediction error - averaged over 100 such evaluation specimens, these errors serve as a metric of ANN performance evaluation.

In Table 5.2, the "One-Plot" ANN uses the points from a single hysteresis plot at one applied stress value. The second ANN, using the "Two-Plot" method, targets the same parameters but uses points from two hysteresis plots corresponding to the two values of stress used in generating the training data-50 and $400 \mathrm{MPa}$. Providing data from two different applied stresses caused a significant increase in characterization accuracy, particularly in the case of stress influence coefficients - a single example illustrating this difference is shown in Table 5.2. This result is as 
Table 5.1: Example SMA Training Specimen Parameters

\begin{tabular}{ccc}
\hline Parameter & Values & Units \\
\hline$T_{0}$ & 165 & ${ }^{\circ} \mathrm{C}$ \\
$E_{A}$ & 63 & $\mathrm{GPa}$ \\
$E_{M}$ & 39 & $\mathrm{GPa}$ \\
$\alpha$ & 0 & $1 /{ }^{\circ} \mathrm{C}$ \\
$M_{s}$ & 54 & ${ }^{\circ} \mathrm{C}$ \\
$M_{f}$ & 20 & ${ }^{\circ} \mathrm{C}$ \\
$A_{s}$ & 55 & ${ }^{\circ} \mathrm{C}$ \\
$A_{f}$ & 95 & ${ }^{\circ} \mathrm{C}$ \\
$C^{M}$ & 15 & $\mathrm{MPa} /{ }^{\circ} \mathrm{C}$ \\
$C^{A}$ & 13 & $\mathrm{MPa} /{ }^{\circ} \mathrm{C}$ \\
$H_{\text {max }}$ & 0.07 & - \\
$H_{\text {min }}$ & 0 & - \\
$k$ & 200 & - \\
$\sigma_{\text {crit }}$ & 0 & $\mathrm{~Pa}$ \\
\hline
\end{tabular}

expected. Because the stress influence coefficients describe how the characteristic transformation temperatures vary with applied stress, it follows that a trained ANN would be incapable of accurately identifying stress influence coefficient values based on strain-temperature data for a single case of constant stress. Because the ANN trained with strain-temperature data at two different cases of constant stress- two hysteresis plots - outperformed the ANN trained with a single hysteresis plot, the two-plot method was adopted for the training of all subsequent ANNs. Including additional hysteresis plots (i.e., switching to a "Three-Plot" or "Four-Plot" method) was left open as a possibility for further ANN performance improvement in the event that the two-plot method did not achieve adequate results. 
Table 5.2: Comparison of 1-Plot and 2-Plot Methods

\begin{tabular}{cccc}
\hline Parameter & Target & 1-Plot & 2-Plot \\
\hline$M_{s}$ & 54 & 50.83 & 54.29 \\
$M_{f}$ & 20 & 14.5 & 20.97 \\
$A_{s}$ & 55 & 50.7 & 56.68 \\
$A_{f}$ & 95 & 91.27 & 95.63 \\
$C^{M}$ & 15 & -1.09 & 14.91 \\
$C^{A}$ & 13 & 13.25 & 13.21 \\
\hline
\end{tabular}

\subsection{Factorial Approach}

ANN performance evaluation was typically performed using 100 randomly generated evaluation specimens, as described. A smaller evaluation set using 20 specimens, however, is given here for ease of presentation. The material parameters corresponding to these 20 specimens are shown in Table 5.3. An example corresponding raw output from a factorial-based ANN is shown in Table 5.4, and the calculated error values are shown in Table 5.5.

As shown in this smaller batch analysis of 20 evaluation specimens, the factorialbased ANN missed the known target values by several orders of magnitude (see Specimens 4 and 9 in Table 5.5) for certain SMA parameter combinations. Tested with one hundred randomly generated SMA specimens, the factorial-based ANN exhibited 13 of these "extreme misses" in which maximum transformation temperature or stress influence coefficient error was greater than $20^{\circ} \mathrm{C}$ or $20 \mathrm{MPa} /{ }^{\circ} \mathrm{C}$ - an "extreme" miss rate of $13 \%$. These cases of abnormally high error are most likely caused by gaps in the training data. Excluding these exceptions, the ANN was generally able to identify transformation temperatures to within $1.04^{\circ} \mathrm{C}$ and stress influence coefficients to within $1.65^{\circ} \mathrm{C} / \mathrm{MPa}$. This level of accuracy was achieved using a training 
Table 5.3: Batch Analysis - Target Values

\begin{tabular}{ccccccc}
\hline Specimen & $M_{s}$ & $M_{f}$ & $A_{s}$ & $A_{f}$ & $C^{M}$ & $C^{A}$ \\
\hline 1 & 78 & 8 & 21 & 103 & 18 & 12 \\
2 & 84 & 14 & 45 & 103 & 10 & 18 \\
3 & 90 & 35 & 68 & 98 & 12 & 15 \\
4 & 45 & 30 & 54 & 91 & 14 & 18 \\
5 & 42 & -3 & 43 & 50 & 16 & 14 \\
6 & 52 & 10 & 32 & 111 & 11 & 10 \\
7 & 59 & 37 & 70 & 90 & 18 & 11 \\
8 & 85 & -9 & 27 & 104 & 14 & 9 \\
9 & 52 & 35 & 42 & 69 & 13 & 9 \\
10 & 27 & -6 & 67 & 87 & 17 & 12 \\
11 & 66 & 40 & 48 & 118 & 8 & 13 \\
12 & 55 & -2 & 66 & 83 & 15 & 16 \\
13 & 30 & 3 & 49 & 98 & 12 & 15 \\
14 & 33 & -14 & 40 & 84 & 12 & 9 \\
15 & 25 & -9 & 60 & 84 & 18 & 15 \\
16 & 46 & -2 & 30 & 69 & 15 & 14 \\
17 & 67 & 28 & 63 & 119 & 11 & 10 \\
18 & 33 & -4 & 29 & 74 & 15 & 16 \\
19 & 56 & 0 & 61 & 78 & 14 & 8 \\
20 & 65 & 11 & 48 & 120 & 8 & 14 \\
\hline & & & & & &
\end{tabular}


Table 5.4: Batch Analysis - Factorial Method (ANN Output Values)

\begin{tabular}{ccccccc}
\hline Specimen & $M_{s}$ & $M_{f}$ & $A_{s}$ & $A_{f}$ & $C^{M}$ & $C^{A}$ \\
\hline 1 & 77.5356 & 7.6420 & 19.2230 & 102.0359 & 17.9173 & 12.8015 \\
2 & 82.4069 & 14.4919 & 45.9348 & 101.8013 & 10.0908 & 12.4323 \\
3 & 87.4238 & 35.9560 & 68.9581 & 91.9614 & 12.3792 & 12.0923 \\
4 & -144.8341 & 50.3385 & 102.4244 & -655.8885 & 20.1915 & -138.6301 \\
5 & 41.7775 & -4.0366 & 43.3027 & 50.6003 & 16.0678 & 13.2927 \\
6 & 51.5100 & 9.9809 & 30.7781 & 110.0420 & 10.7960 & 13.1468 \\
7 & 58.9088 & 37.3274 & 69.9380 & 89.7062 & 18.0533 & 13.4324 \\
8 & 84.6202 & -10.4135 & 25.1641 & 103.5695 & 13.3245 & 13.1374 \\
9 & -140.5437 & 54.9426 & 89.7058 & -680.1467 & 19.4533 & -139.1184 \\
10 & 27.5715 & -7.1176 & 67.7416 & 88.5811 & 16.6024 & 13.9818 \\
11 & 62.8361 & 38.0186 & 48.8641 & 114.6972 & 8.1655 & 21.0444 \\
12 & 54.9837 & -2.6804 & 66.5288 & 84.0590 & 15.1874 & 12.1992 \\
13 & 30.3963 & 2.6441 & 50.2623 & 99.0943 & 12.0049 & 13.0181 \\
14 & 33.1791 & -14.7512 & 39.8708 & 84.3980 & 11.9289 & 12.8721 \\
15 & 25.6396 & -9.9084 & 61.6171 & 85.4343 & 17.9724 & 13.0277 \\
16 & 45.2571 & -3.1009 & 28.3134 & 68.5780 & 14.6886 & 12.2131 \\
17 & 65.0175 & 28.4829 & 63.8876 & 116.2291 & 10.6797 & 12.0985 \\
18 & 32.0677 & -5.2904 & 27.2349 & 72.8780 & 14.5367 & 11.3868 \\
19 & 56.7470 & -0.9313 & 62.3944 & 80.0466 & 13.6880 & 13.6915 \\
20 & 64.0017 & 10.9126 & 49.0661 & 118.7610 & 8.2612 & 11.9102 \\
\hline
\end{tabular}


Table 5.5: Batch Analysis - Factorial Method (Error Values)

\begin{tabular}{ccccccc}
\hline Specimen & $M_{s}$ & $M_{f}$ & $A_{s}$ & $A_{f}$ & $C^{M}$ & $C^{A}$ \\
\hline 1 & 0.4644 & 0.3580 & 1.7770 & 0.9641 & 0.0827 & 0.8015 \\
2 & 1.5931 & 0.4919 & 0.9348 & 1.1987 & 0.0908 & 5.5677 \\
3 & 2.5762 & 0.9560 & 0.9581 & 6.0386 & 0.3792 & 2.9077 \\
4 & 189.8341 & 20.3385 & 48.4244 & 746.8885 & 6.1915 & 156.6301 \\
5 & 0.2225 & 1.0366 & 0.3027 & 0.6003 & 0.0678 & 0.7073 \\
6 & 0.4900 & 0.0191 & 1.2219 & 0.9580 & 0.2040 & 3.1468 \\
7 & 0.0912 & 0.3274 & 0.0620 & 0.2938 & 0.0533 & 2.4324 \\
8 & 0.3798 & 1.4135 & 1.8359 & 0.4305 & 0.6755 & 4.1374 \\
9 & 192.5437 & 19.9426 & 47.7058 & 749.1467 & 6.4533 & 148.1184 \\
10 & 0.5715 & 1.1176 & 0.7416 & 1.5811 & 0.3976 & 1.9818 \\
11 & 3.1639 & 1.9814 & 0.8641 & 3.3028 & 0.1655 & 8.0444 \\
12 & 0.0163 & 0.6804 & 0.5288 & 1.0590 & 0.1874 & 3.8008 \\
13 & 0.3963 & 0.3559 & 1.2623 & 1.0943 & 0.0049 & 1.9819 \\
14 & 0.1791 & 0.7512 & 0.1292 & 0.3980 & 0.0711 & 3.8721 \\
15 & 0.6396 & 0.9084 & 1.6171 & 1.4343 & 0.0276 & 1.9723 \\
16 & 0.7429 & 1.1009 & 1.6866 & 0.4220 & 0.3114 & 1.7869 \\
17 & 1.9825 & 0.4829 & 0.8876 & 2.7709 & 0.3203 & 2.0985 \\
18 & 0.9323 & 1.2904 & 1.7651 & 1.1220 & 0.4633 & 4.6132 \\
19 & 0.7470 & 0.9313 & 1.3944 & 2.0466 & 0.3120 & 5.6915 \\
20 & 0.9983 & 0.0874 & 1.0661 & 1.2390 & 0.2612 & 2.0898 \\
\hline AVG & 19.9282 & 2.7286 & 5.7583 & 76.1494 & 0.8360 & 18.1191 \\
\hline AVG* & $\mathbf{0 . 8 9 9 3}$ & $\mathbf{0 . 7 9 3 9}$ & $\mathbf{1 . 0 5 7 5}$ & $\mathbf{1 . 4 9 7 4}$ & $\mathbf{0 . 2 2 6 4}$ & $\mathbf{3 . 2 0 1 9}$ \\
& & & & & & \\
& $*$ Excluding "Extreme Misses" - Specimens 4 and 9 &
\end{tabular}


Table 5.6: ANN Performance Comparison: Factorial Approach

\begin{tabular}{rrr} 
& Factorial-360 & Factorial-4296 \\
\hline Training SMA specimens: & 360 & 4296 \\
"Extreme Misses": & $77 \%$ & $13 \%$ \\
Transformation temperature: "Hits": & $11 \%$ & $74 \%$ \\
Mean error: & $14.04{ }^{\circ} \mathrm{C}$ & $4.39^{\circ} \mathrm{C}$ \\
Standard deviation: & $10.32{ }^{\circ} \mathrm{C}$ & $12.86{ }^{\circ} \mathrm{C}$ \\
Stress influence coefficient: "Hits": & $37 \%$ & $74 \%$ \\
Mean error: & $16.39 \mathrm{MPa} /{ }^{\circ} \mathrm{C}$ & $2.52 \mathrm{MPa} /{ }^{\circ} \mathrm{C}$ \\
Standard deviation: & $11.14 \mathrm{MPa} /{ }^{\circ} \mathrm{C}$ & $2.74 \mathrm{MPa} /{ }^{\circ} \mathrm{C}$ \\
\hline
\end{tabular}

data set consisting of 4296 samples.

A comparison of ANN performance for factorial-based ANNs using 360 and 4296 training specimens (with parameters corresponding to Tables 4.1 and 4.2, respectively) is summarized in Figure 5.2 and Table 5.6. Figure 5.2 gives a histogram of transformation and stress influence coefficient errors for the two trained ANNs, and Table 5.6 provides quantitative performance metrics. Instances in which the ANN identifies a transformation temperature within $3^{\circ} \mathrm{C}$ or a stress influence coefficient within $3 \mathrm{MPa} /{ }^{\circ} \mathrm{C}$ are classified as "hits". Note that in this comparison, to allow fair comparison, both ANNs were evaluated using the same set of 100 randomly generated evaluation specimens. As expected, increasing the amount of training data yields increased ANN material parameter identification accuracy. This increase in performance can be seen both qualitatively in Figure 5.2 and quantitatively through the decreases in "extreme misses", transformation temperature and stress influence coefficient mean error, and increases in transformation and stress influence coefficient "hit" rates. 

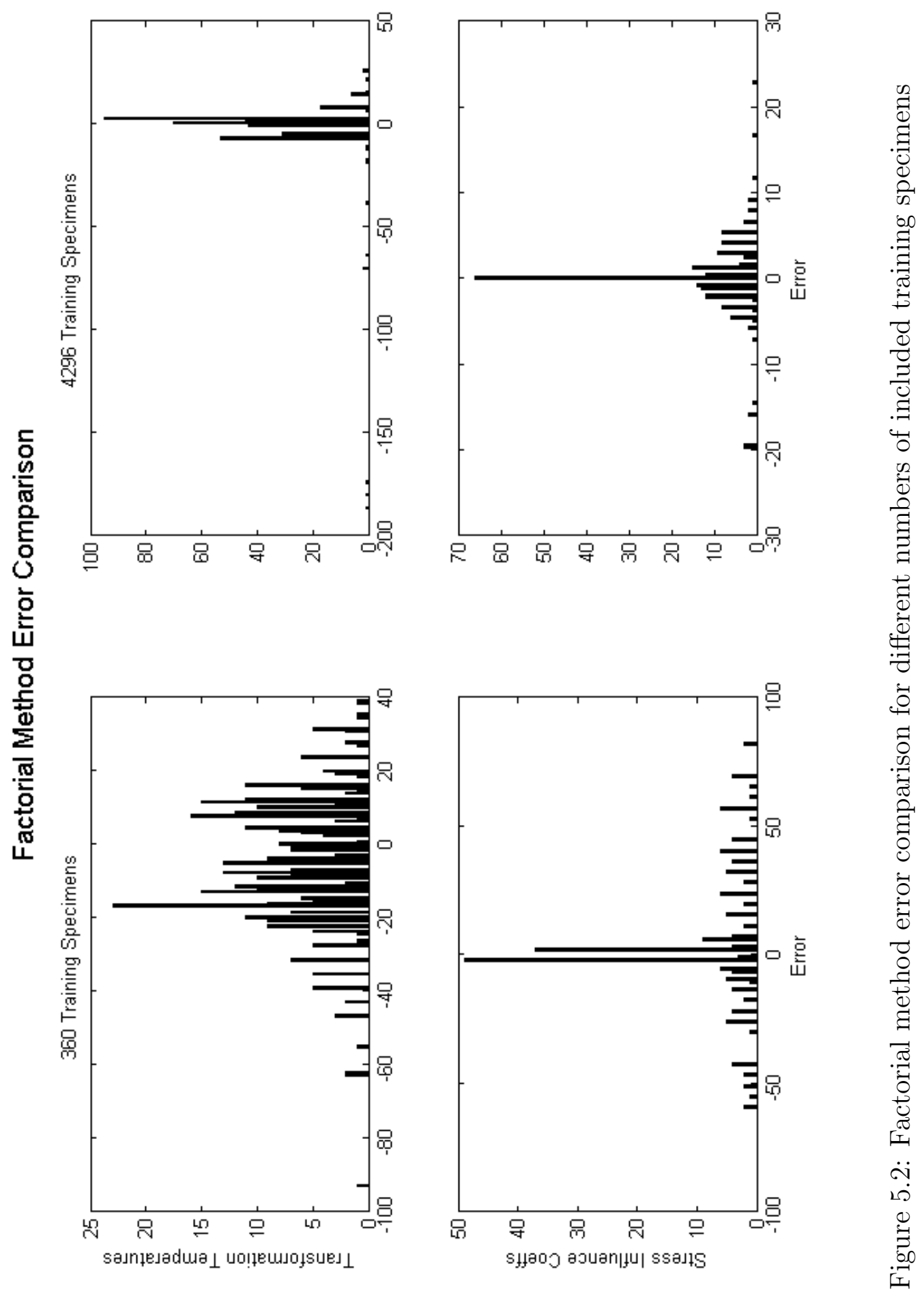


\subsection{Taguchi-Factorial Approach}

The performance evaluation process was then repeated for the Taguchi-Factorialbased ANN. Results corresponding to the 20 test specimens listed in Table 5.3 are shown in Tables 5.7 and 5.8. The raw output of a Taguchi-based ANN trained with 482 training specimens is shown in Table 5.7, and corresponding error values are given in Table 5.8. Compared with the batch results presented in Section 5.3, it is evident that the Taguchi-based approach largely eliminates the previously observed "extreme misses". A comparison of two Taguchi-based ANNs trained using differing numbers of training specimens (26 and 482 as specified in Tables 4.6 and 4.7) is given. Again, for the sake of fair comparison, the two ANNs are evaluated using the same set of 100 randomly generated evaluation specimens. As shown, the Taguchi-based ANN is able to achieve transformation temperature and stress influence coefficient "hit" rates of $83 \%$ and $69 \%$, respectively, with only 482 training specimens.

To further assess the Taguchi-based approach to training specimen parameter selection, a few Taguchi-based ANNs were compared with ANNs trained using randombased training data - that is, training specimens in which parameter values were randomly selected between specified bounds (see Table 4.1). An illustrative comparison is shown in Figure 5.4 and Table 5.10. With the same number of training specimens, and with the same range of parameter values, the Taguchi-based ANN outperforms the random-based ANN. This result highlights the Taguchi method capability of efficiently exploring system dynamics with relatively few system parameter varia-

tions. A Taguchi-based approach to training specimen parameter variation offers additional advantages over a random-based approach by allowing manual selection and adjustment of possible parameter values, and by yielding repeatable results. These advantages allow more precise tuning of the training data and could play a 
Table 5.7: Batch Analysis - Taguchi-Factorial Method (ANN Output Values)

\begin{tabular}{ccccccc}
\hline Specimen & $M_{s}$ & $M_{f}$ & $A_{s}$ & $A_{f}$ & $C^{M}$ & $C^{A}$ \\
\hline 1 & 78.1303 & 7.4996 & 20.8754 & 103.0814 & 17.9399 & 5.4790 \\
2 & 84.2341 & 12.8727 & 44.7590 & 102.8838 & 9.8274 & 3.6995 \\
3 & 91.0791 & 29.5502 & 66.7531 & 98.0555 & 12.1734 & 8.1781 \\
4 & 45.6356 & 26.8683 & 53.2224 & 91.0092 & 14.2022 & 7.1486 \\
5 & 42.0519 & -3.0626 & 43.0208 & 49.9949 & 16.5108 & 16.3444 \\
6 & 52.0949 & 9.3438 & 31.8037 & 110.8673 & 11.2639 & 7.8072 \\
7 & 60.1139 & 31.6781 & 68.7637 & 90.0177 & 18.2426 & 11.0646 \\
8 & 84.9574 & -9.0424 & 26.9591 & 103.9642 & 13.8277 & 5.5185 \\
9 & 52.9069 & 30.1857 & 40.7656 & 69.0900 & 12.6538 & 14.1816 \\
10 & 27.0020 & -6.0297 & 66.9443 & 86.9266 & 16.3089 & 17.8382 \\
11 & 67.3900 & 33.8060 & 46.4971 & 118.1439 & 7.1343 & 12.0845 \\
12 & 54.9956 & -1.9923 & 66.0140 & 82.9940 & 14.9739 & 19.1651 \\
13 & 30.0553 & 2.7629 & 48.9576 & 97.8672 & 12.2250 & 9.9819 \\
14 & 32.9761 & -14.0112 & 39.9758 & 84.0420 & 11.7321 & 13.9515 \\
15 & 24.9851 & -9.0677 & 60.0089 & 83.8241 & 17.1087 & 6.5454 \\
16 & 45.9751 & -2.0489 & 29.9444 & 69.0300 & 14.6985 & 18.3696 \\
17 & 67.7070 & 24.9342 & 62.3100 & 119.0157 & 10.7498 & 6.8536 \\
18 & 32.9624 & -3.9253 & 28.9678 & 73.9319 & 14.4981 & 14.5684 \\
19 & 55.9776 & -0.1233 & 60.9520 & 77.9799 & 13.8962 & 14.5915 \\
20 & 65.1664 & 10.3167 & 47.8850 & 119.7794 & 7.7119 & 7.7290 \\
\hline
\end{tabular}


Table 5.8: Batch Analysis - Taguchi-Factorial Method (Error Values)

\begin{tabular}{ccccccc}
\hline Specimen & $M_{s}$ & $M_{f}$ & $A_{s}$ & $A_{f}$ & $C^{M}$ & $C^{A}$ \\
\hline 1 & 0.1303 & 0.5004 & 0.1246 & 0.0814 & 0.0601 & 6.5210 \\
2 & 0.2341 & 1.1273 & 0.2410 & 0.1162 & 0.1726 & 14.3005 \\
3 & 1.0791 & 5.4498 & 1.2469 & 0.0555 & 0.1734 & 6.8219 \\
4 & 0.6356 & 3.1317 & 0.7776 & 0.0092 & 0.2022 & 10.8514 \\
5 & 0.0519 & 0.0626 & 0.0208 & 0.0051 & 0.5108 & 2.3444 \\
6 & 0.0949 & 0.6562 & 0.1963 & 0.1327 & 0.2639 & 2.1928 \\
7 & 1.1139 & 5.3219 & 1.2363 & 0.0177 & 0.2426 & 0.0646 \\
8 & 0.0426 & 0.0424 & 0.0409 & 0.0358 & 0.1723 & 3.4815 \\
9 & 0.9069 & 4.8143 & 1.2344 & 0.0900 & 0.3462 & 5.1816 \\
10 & 0.0020 & 0.0297 & 0.0557 & 0.0734 & 0.6911 & 5.8382 \\
11 & 1.3900 & 6.1940 & 1.5029 & 0.1439 & 0.8657 & 0.9155 \\
12 & 0.0044 & 0.0077 & 0.0140 & 0.0060 & 0.0261 & 3.1651 \\
13 & 0.0553 & 0.2371 & 0.0424 & 0.1328 & 0.2250 & 5.0181 \\
14 & 0.0239 & 0.0112 & 0.0242 & 0.0420 & 0.2679 & 4.9515 \\
15 & 0.0149 & 0.0677 & 0.0089 & 0.1759 & 0.8913 & 8.4546 \\
16 & 0.0249 & 0.0489 & 0.0556 & 0.0300 & 0.3015 & 4.3696 \\
17 & 0.7070 & 3.0658 & 0.6900 & 0.0157 & 0.2502 & 3.1464 \\
18 & 0.0376 & 0.0747 & 0.0322 & 0.0681 & 0.5019 & 1.4316 \\
19 & 0.0224 & 0.1233 & 0.0480 & 0.0201 & 0.1038 & 6.5915 \\
20 & 0.1664 & 0.6833 & 0.1150 & 0.2206 & 0.2881 & 6.2710 \\
\hline AVG & $\mathbf{0 . 3 3 6 9}$ & $\mathbf{1 . 5 8 2 5}$ & $\mathbf{0 . 3 8 5 4}$ & $\mathbf{0 . 0 7 3 6}$ & $\mathbf{0 . 3 2 7 8}$ & $\mathbf{5 . 0 9 5 6}$ \\
\hline
\end{tabular}

Table 5.9: ANN Performance Comparison: Taguchi-Factorial Approach

\begin{tabular}{rrr} 
& Taguchi-26 & Taguchi- 482 \\
\hline Training SMA specimens: & 26 & 482 \\
"Extreme Misses": & $8 \%$ & $0 \%$ \\
Transformation temperature: "Hits": & $25 \%$ & $83 \%$ \\
Mean error: & $7.2^{\circ} \mathrm{C}$ & $1.66^{\circ} \mathrm{C}$ \\
Standard deviation: & $5.49^{\circ} \mathrm{C}$ & $1.32^{\circ} \mathrm{C}$ \\
Stress influence coefficient: "Hits": & $62 \%$ & $69 \%$ \\
Mean error: & $2.90 \mathrm{MPa} /{ }^{\circ} \mathrm{C}$ & $2.75 \mathrm{MPa} /{ }^{\circ} \mathrm{C}$ \\
Standard deviation: & $2.06 \mathrm{MPa} /{ }^{\circ} \mathrm{C}$ & $2.00 \mathrm{MPa} /{ }^{\circ} \mathrm{C}$ \\
\hline
\end{tabular}



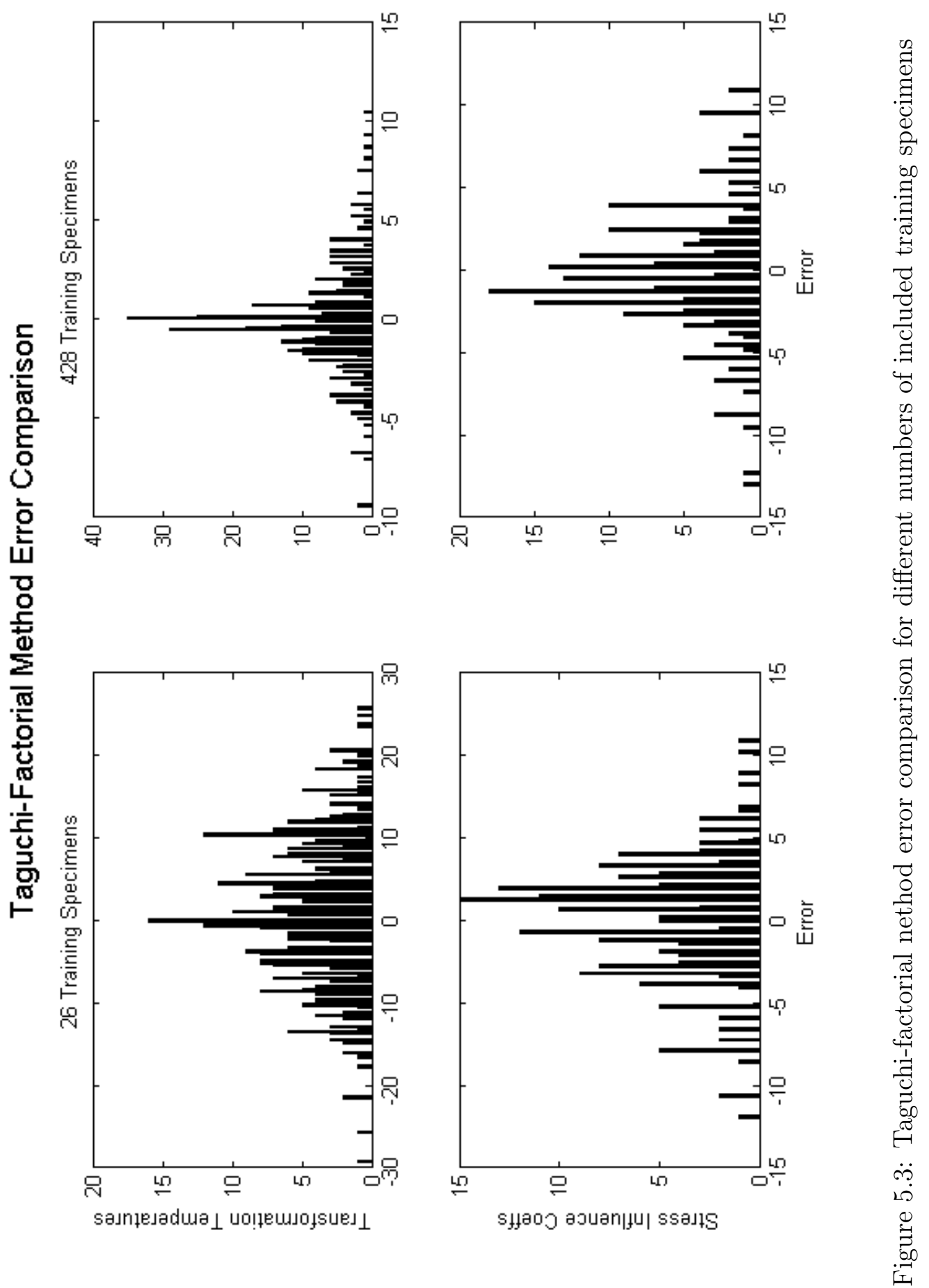
Table 5.10: ANN Performance Comparison: Random vs Taguchi-Factorial Methods

\begin{tabular}{rrr} 
& Random & Taguchi-Factorial \\
\hline Training SMA specimens: & 26 & 26 \\
"Extreme Misses": & $53 \%$ & $11 \%$ \\
Transformation temperature: "Hits": & $18 \%$ & $28 \%$ \\
Mean error: & $14.39^{\circ} \mathrm{C}$ & $7.60^{\circ} \mathrm{C}$ \\
Standard deviation: & $14.80^{\circ} \mathrm{C}$ & $8.01{ }^{\circ} \mathrm{C}$ \\
Stress influence coefficient: "Hits": & $52 \%$ & $62 \%$ \\
Mean error: & $4.16 \mathrm{MPa} /{ }^{\circ} \mathrm{C}$ & $3.05 \mathrm{MPa} /{ }^{\circ} \mathrm{C}$ \\
Standard deviation: & $3.83 \mathrm{MPa} /{ }^{\circ} \mathrm{C}$ & $2.47 \mathrm{MPa} /{ }^{\circ} \mathrm{C}$ \\
\hline
\end{tabular}

significant role in more advanced implementations of an ANN-based approach to SMA material parameter identification.

A direct comparison between ANNs trained using factorial and Taguchi-based training data is summarized with Figure 5.5 and Table 5.11. Again, to more fairly compare overall results from the factorial and Taguchi-based ANNs, performance evaluation performed by assessing each ANN with the same set of 100 randomly generated evaluation specimens. Figure 5.5 provides an error histogram comparison, and Table 5.11 provides comparison of performance values. These results both qualitatively and quantitatively show that the Taguchi-based ANN clearly outperforms the factorial-based ANN despite using significantly less training data. This can be attributed to the fact that the Taguchi-based training data contained more variety in its SMA parameter values. 

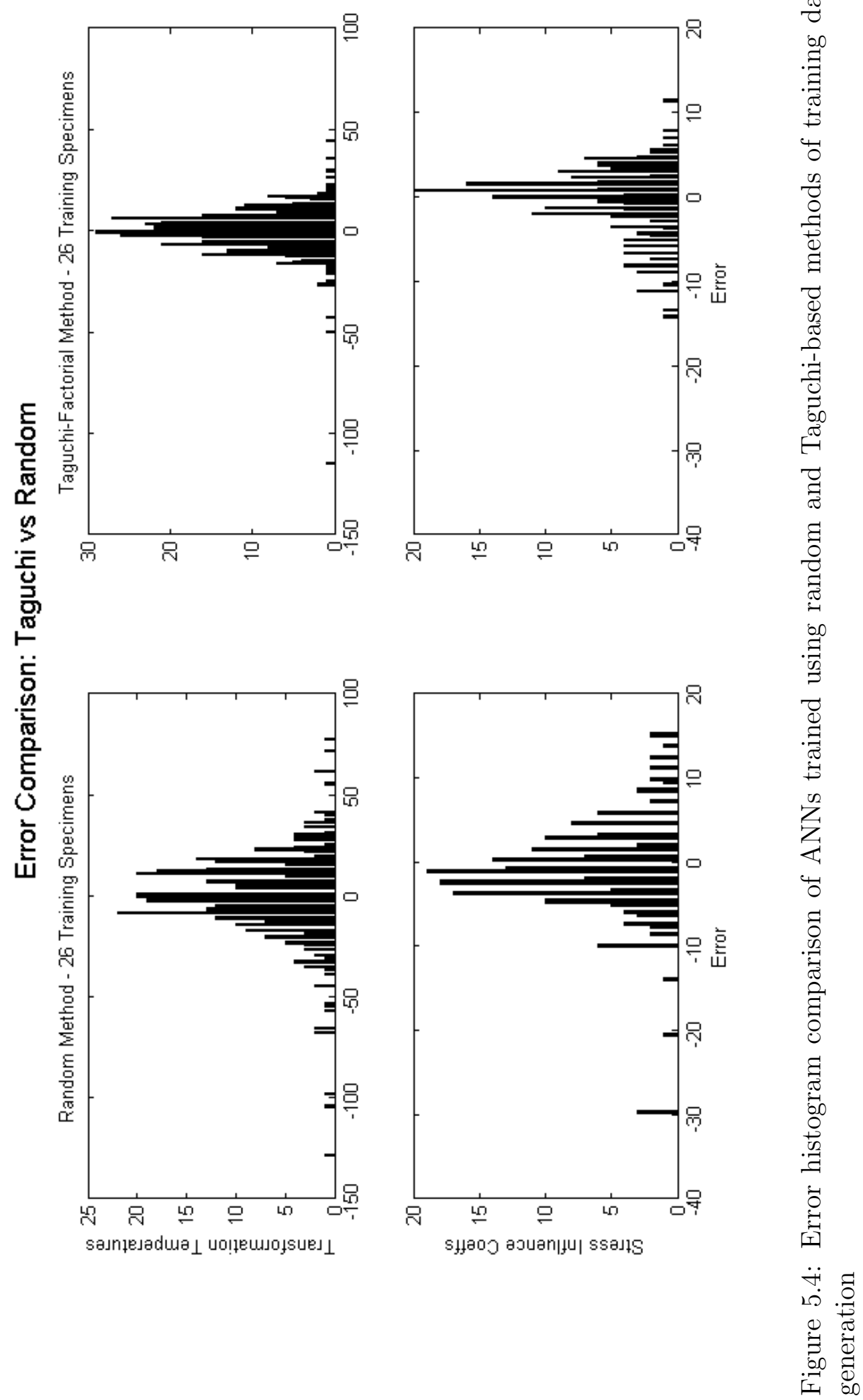

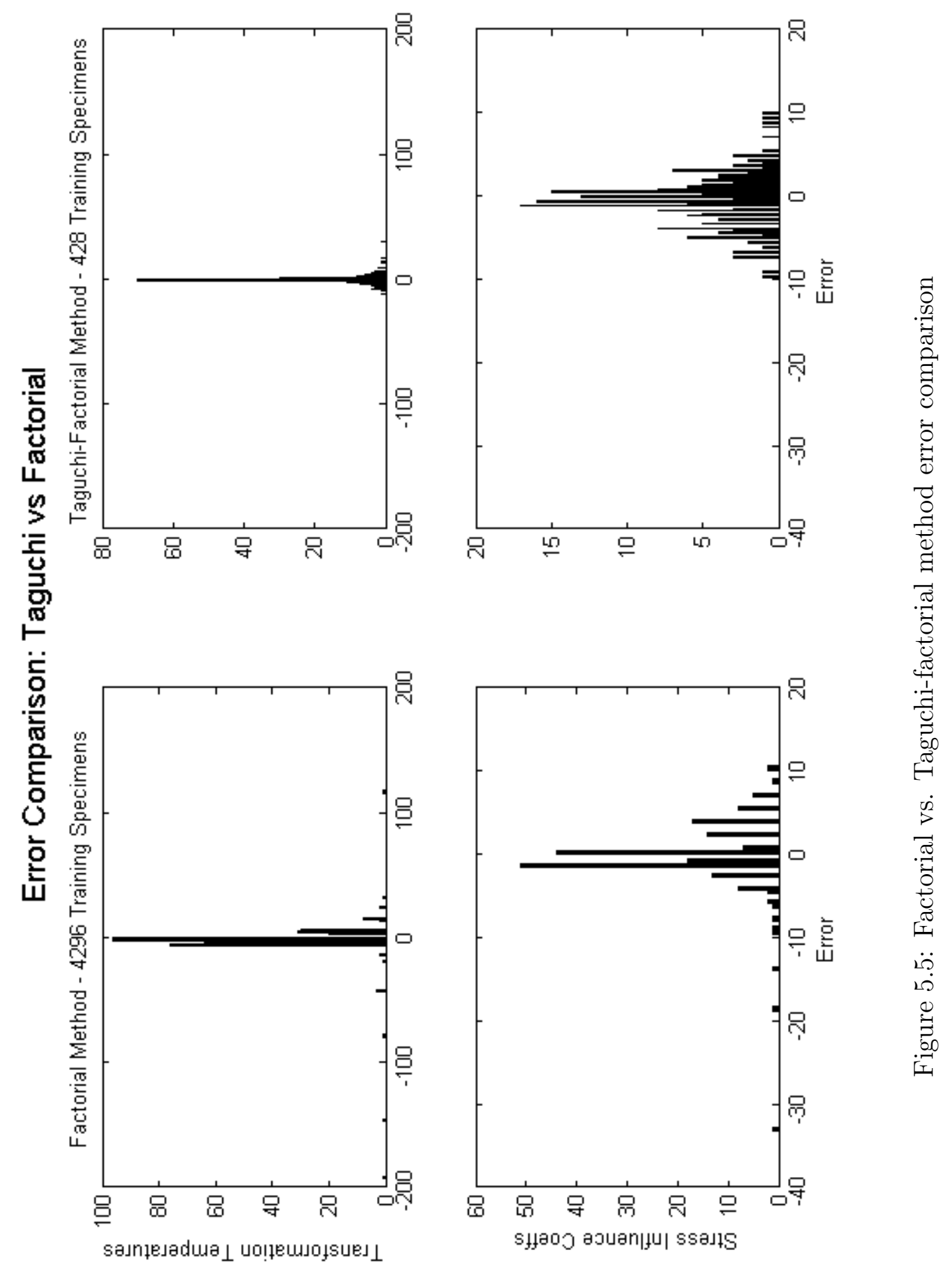
Table 5.11: ANN Performance Comparison: Taguchi vs. Factorial

\begin{tabular}{rrr} 
& Factorial & Taguchi-Factorial \\
\hline Training SMA specimens: & 4296 & 482 \\
"Extreme Misses": & $8 \%$ & $1 \%$ \\
Transformation temperature: "Hits": & $75 \%$ & $87 \%$ \\
Mean error: & $3.9^{\circ} \mathrm{C}$ & $1.59^{\circ} \mathrm{C}$ \\
Standard deviation: & $11.27^{\circ} \mathrm{C}$ & $2.12^{\circ} \mathrm{C}$ \\
Stress influence coefficient: "Hits": & $73 \%$ & $72 \%$ \\
Mean error: & $2.38 \mathrm{MPa} /{ }^{\circ} \mathrm{C}$ & $2.34 \mathrm{MPa} /{ }^{\circ} \mathrm{C}$ \\
Standard deviation: & $2.97 \mathrm{MPa} /{ }^{\circ} \mathrm{C}$ & $1.73 \mathrm{MPa} /{ }^{\circ} \mathrm{C}$ \\
\hline
\end{tabular}




\section{CONCLUSIONS AND RECOMMENDATIONS}

Based on the presented results, a number of conclusions and recommendations are made.

\subsection{Conclusions}

- It is evident that the developed approach yields ANNs largely capable of identifying SMA material parameters-the transformation temperatures $M_{S}, M_{F}$, $A_{S}, A_{F}$, and the stress influence coefficients $C^{M}$ and $C^{A}$. Different approaches for the generation of training data specimens required different total numbers of training specimens for comparable ANN performance, but each approach led to an ANN that could, for the most part, successfully identify values for these SMA material parameters.

- Of the approaches assessed for training specimen generation, the one implementing Taguchi methods was found to be significantly more capable of yielding a high-performance ANN with a limited number of training specimens. Using 428 training specimens, the Taguchi-based approach yielded an ANN that outperformed competing ANNs trained using 4296 specimens.

- The developed method is judged to be a feasible and promising candidate for shape memory alloy characterization. Once trained, an ANN is shown to be capable of accurately identifying SMA parameter values for a given specimen using only two sets of strain-temperature hysteresis data. By using less straintemperature data and necessitating relatively little data processing, this approach potentially provides a more rapid approach to SMA material parameter identification than existing characterization methods. 


\subsection{Recommendations}

Future work seeking to further refine this methodology could focus on one of several areas.

- The constitutive model-based training data used in this work could be supplemented with real-world, experimentally-derived SMA strain-temperature data. Furthermore, if the experimental SMA data required for this task is made available, ANN performance assessment could be repeated using the real-world data.

- Now that an overall methodology has been established and demonstrated, further attention could be focused towards the structure and training of the ANN - whether this involves the development of a custom ANN training algorithm or simply a more detailed analysis of the different MATLAB ANN toolbox options.

- To facilitate additional performance analysis and to improve practical usability, it could be worthwhile to implement some form of uncertainty quantification; that is, it would be desirable for the trained ANN to output material parameter values as well as estimated error bounds or some other indication of relative certainty. One possible way of achieving at least a "relative certainty" indication for a given SMA specimen would be to compare its given strain-temperature coordinates with the training data. If the coordinates are similar to that of a training specimen, the ANN output would have a relatively high level of certainty. If there are no similar matches in the training data, the ANN output would have a lower level of certainty.

- Future work could focus on including variation of additional SMA material 
properties in the generation of training data. As a feasibility study, a few parameters, such as $\alpha, H_{m i n}, k$, and $\sigma_{c r i t}$, have been fixed at constant values throughout this work. With feasibility having now been demonstrated for this methodology, increasing the range of applicability is a logical continuation.

- Future work enabled by implementation of an SMA characterizing ANN generated using the methods developed in this thesis could possibly include more advanced study of SMA material parameters. For example, the ability to rapidly determine characteristic SMA material properties could facilitate studies focused on modeling the evolution of SMA material parameter values over material lifetime and long-term loading cycles or on investigating how SMA material parameter values vary with different manufacturing processes or heat treatments. 


\section{REFERENCES}

[1] Christopher M Bishop. Pattern recognition and machine learning, Volume 1. Springer, New York, 2006.

[2] James G Boyd and Dimitris C Lagoudas. A thermodynamical constitutive model for shape memory materials. Part I. The monolithic shape memory alloy. International Journal of Plasticity, 12(6):805-842, 1996.

[3] J Burpee, T Duerig, and D Stockel. Shape memory alloy stent, October 222003. EP Patent 0,873,734.

[4] CB Churchill, JA Shaw, and MA Iadicola. Tips and tricks for characterizing shape memory alloy wire: part 2fundamental isothermal responses. Experimental Techniques, 33(1):51-62, 2009.

[5] Lawrence Davis. Handbook of genetic algorithms. Van Nostrand Reinhold, New York, 1991.

[6] Howard Demuth, Mark Beale, and Math Works. MATLAB: Neural Network Toolbox: User's Guide. Math Works, 1992.

[7] Richard M Friedberg. A learning machine: Part i. IBM Journal of Research and Development, 2(1):2-13, 1958.

[8] JA Ghani, IA Choudhury, and HH Hassan. Application of taguchi method in the optimization of end milling parameters. Journal of Materials Processing Technology, 145(1):84-92, 2004.

[9] David Edward Goldberg. Genetic algorithms in search, optimization, and machine learning, volume 412. Addison-Wesley: Menlo Park, California, 1989. 
[10] MT Hagan, HB Demuth, and MH Beale. Neural network design. Pws Pub. Boston, MA, 1996.

[11] Darren J Hartl. Modeling of shape memory alloys considering rate-independent and rate-dependent irrecoverable strains. PhD Dissertation, Texas A\&M University, 2010.

[12] Darren J Hartl and Dimitris C Lagoudas. Characterization and 3-d modeling of ni60ti sma for actuation of a variable geometry jet engine chevron. In The 14 th International Symposium on: Smart Structures and Materials \& Nondestructive Evaluation and Health Monitoring, San Diego, CA, pages 65293Z-65293Z. International Society for Optics and Photonics, 2007.

[13] DJ Hartl, DC Lagoudas, and FT Calkins. Advanced methods for the analysis, design, and optimization of sma-based aerostructures. Smart Materials and Structures, 20(9):094006, 2011.

[14] Darren J Hartl and Dimitris C Lagoudas. Aerospace applications of shape memory alloys. Proceedings of the Institution of Mechanical Engineers, Part G: Journal of Aerospace Engineering, 221(4):535-552, 2007.

[15] DJ Hartl, JT Mooney, DC Lagoudas, FT Calkins, and JH Mabe. Use of a ni60ti shape memory alloy for active jet engine chevron application: Ii. experimentally validated numerical analysis. Smart Materials and Structures, 19(1):015021, 2010.

[16] Mohamad H Hassoun. Fundamentals of artificial neural networks. MIT press: Cambridge, MA, 1995.

[17] LP Kaelbling, ML Littman, and AW Moore. Reinforcement learning: A survey. arXiv preprint cs/9605103, 1996. 
[18] A Kilicarslan, G Song, and KM Grigoriadis. Modeling and hysteresis compensation in a thin sma wire using anfis methods. Journal of Intelligent Material Systems and Structures, 22(1):45-57, 2011.

[19] B Kim, MG Lee, YP Lee, Y Kim, and G Lee. An earthworm-like micro robot using shape memory alloy actuator. Sensors and Actuators A: Physical, 125(2):429-437, 2006.

[20] K Kirkpatrick, J May Jr, and J Valasek. Aircraft system identification using artificial neural networks. In 51st AIAA Aerospace Sciences Meeting including the New Horizons Forum and Aerospace Exposition, Grapevine, TX, 2013.

[21] Kenton Kirkpatrick and John Valasek. Reinforcement learning for characterizing hysteresis behavior of shape memory alloys. Journal of Aerospace Computing, Information, and Communication, 6(3):227-238, 2009.

[22] Kenton Kirkpatrick and John Valasek. Active length control of shape memory alloy wires using reinforcement learning. Journal of Intelligent Material Systems and Structures, 22(14):1595-1604, 2011.

[23] Kenton Conrad Kirkpatrick. Reinforcement learning for active length control and hysteresis characterization of shape memory alloys. PhD Dissertation, Texas A\&M University, 2009.

[24] D Lagoudas, D Hartl, Y Chemisky, L Machado, and P Popov. Constitutive model for the numerical analysis of phase transformation in polycrystalline shape memory alloys. International Journal of Plasticity, 32:155-183, 2012.

[25] Dimitris C Lagoudas. Shape memory alloys: modeling and engineering applications. Springer, New York, 2008. 
[26] Y LeCun, B Boser, JS Denker, D Henderson, RE Howard, et al. Backpropagation applied to handwritten zip code recognition. Neural Computation, 1(4):541$551,1989$.

[27] C Liang and CA Rogers. A multi-dimensional constitutive model for shape memory alloys. Journal of Engineering Mathematics, 26(3):429-443, 1992.

[28] N Ma, G Song, and HJ Lee. Position control of shape memory alloy actuators with internal electrical resistance feedback using neural networks. Smart Materials and Structures, 13(4):777, 2004.

[29] K Mehrotra, CK Mohan, and S Ranka. Elements of artificial neural networks. MIT press, Cambridge, MA, 1997.

[30] Tom M Mitchell. Machine learning. 1997. McGraw Hill, Burr Ridge, IL, 1997.

[31] HA Rowley, S Baluja, and T Kanade. Neural network-based face detection. Pattern Analysis and Machine Intelligence, IEEE Transactions on, 20(1):2338, 1998.

[32] Ranjit K Roy. A primer on the Taguchi method. Van Nostrand Reinhold, New York, 1990.

[33] JA Shaw, CB Churchill, and MA Iadicola. Tips and tricks for characterizing shape memory alloy wire: part 1 differential scanning calorimetry and basic phenomena. Experimental Techniques, 32(5):55-62, 2008.

[34] G Song, V Chaudhry, and C Batur. Precision tracking control of shape memory alloy actuators using neural networks and a sliding-mode based robust controller. Smart Materials and Structures, 12(2):223, 2003.

[35] Richard S Sutton and Andrew G Barto. Introduction to reinforcement learning. MIT Press, Cambridge, MA, 1998. 
[36] Genichi Taguchi. System of experimental design: engineering methods to optimize quality and minimize costs, volume 1. UNIPUB/Kraus International Publications, White Plains, NY, 1987.

[37] W Grey Walter. A machine that learns. Scientific American, 185(2):60-63, 1951.

[38] WH Yang and YS Tarng. Design optimization of cutting parameters for turning operations based on the taguchi method. Journal of Materials Processing Technology, 84(1):122-129, 1998. 